Draft version November 19, 2018

Preprint typeset using IATEX style emulateapj v. 8/13/10

\title{
WAVE PROPAGATION AND JET FORMATION IN THE CHROMOSPHERE
}

\author{
L. HEGGLAND \\ Institute of Theoretical Astrophysics, University of Oslo, P.O. Box 1029, Blindern, N-0315 Oslo, Norway \\ V. H. HANSTEEN ${ }^{1}$ \\ Institute of Theoretical Astrophysics, University of Oslo, P.O. Box 1029, Blindern, N-0315 Oslo, Norway
}

\author{
B. De Pontieu \\ Lockheed Martin Solar and Astrophysics Laboratory, 3251 Hanover Street, Org. ADBS, Building 252, Palo Alto, CA 94304 \\ AND \\ M. CARLSSON ${ }^{1}$ \\ Institute of Theoretical Astrophysics, University of Oslo, P.O. Box 1029, Blindern, N-0315 Oslo, Norway \\ Draft version November 19, 2018
}

\begin{abstract}
We present the results of numerical simulations of wave propagation and jet formation in solar atmosphere models with different magnetic field configurations. The presence in the chromosphere of waves with periods longer than the acoustic cutoff period has been ascribed to either strong inclined magnetic fields, or changes in the radiative relaxation time. Our simulations include a sophisticated treatment of radiative losses, as well as fields with different strengths and inclinations. Using Fourier and wavelet analysis techniques, we investigate the periodicity of the waves that travel through the chromosphere. We find that the velocity signal is dominated by waves with periods around 5 minutes in regions of strong, inclined field, including at the edges of strong flux tubes where the field expands, whereas 3-minute waves dominate in regions of weak or vertically oriented fields. Our results show that the field inclination is very important for long-period wave propagation, whereas variations in the radiative relaxation time have little effect. Furthermore, we find that atmospheric conditions can vary significantly on timescales of a few minutes, meaning that a Fourier analysis of wave propagation can be misleading. Wavelet techniques take variations with time into account and are more suitable analysis tools. Finally, we investigate the properties of jets formed by the propagating waves once they reach the transition region, and find systematic differences between the jets in inclined field regions and those in vertical field regions, in agreement with observations of dynamic fibrils.
\end{abstract}

Subject headings: MHD — Sun: atmospheric motions — Sun: chromosphere — Sun: magnetic fields - Sun: transition region

\section{INTRODUCTION}

Chromospheric wave propagation has been an extensively studied, but poorly understood, subject in solar physics for some time. One puzzle has been the presence of propagating waves with periods on the order of 5 minutes or more. Such waves were observed by, e.g., Orrall (1966) and Giovanelli et al. (1978); this was considered surprising since the acoustic cutoff period in the chromosphere, above which waves should not be able to propagate, is on the order of $200 \mathrm{~s}$. Such long-period propagation was later found to be widespread, generally occurring wherever the local magnetic field is strong. Lites et al. (1993) show an example of observations of neighboring internetwork and network regions that have a marked difference in their Fourier spectra.

It was realized (Michalitsanos 1973; Bel \& Lerov 1977; Suematsu 1990) that, since magnetoacoustic waves in a strongly magnetized medium are restricted to propagating along field lines, the effective gravity (i.e., the com-

lars.heggland@astro.uio.no

${ }^{1}$ Also at Center of Mathematics for Applications, University of Oslo, P.O. Box 1053, Blindern, N-0316 Oslo, Norway ponent of gravity along the magnetic field) would be reduced in magnetic regions. Since the cutoff frequency (in an isothermal atmosphere) is given by

$$
\nu_{a c}=\frac{\gamma g_{e f f}}{4 \pi c_{s}}
$$

where $c_{s}$ is the sound speed and $\gamma$ is the ratio of specific heats, the cutoff frequency would also be lower in regions of strong inclined field, potentially allowing 5-minute $(\sim 3 \mathrm{mHz})$ waves to propagate. This hypothesis has since been tested in a number of increasingly advanced numerical simulations, e.g. De Pontieu et al. (2004), Hansteen et al. (2006), and Heggland et al. (2007). All have found that it is an effective mechanism for transmitting 5-minute power through the chromosphere; some models have suggested that the leakage of 5-minute waves into the chromosphere can also explain the presence of 5minute oscillatory signal in the corona (De Pontieu et al. 2005).

One criticism of this explanation has been that not all observations of 5-minute propagation are in regions of obviously inclined field. An alternative explanation has therefore been suggested, originally by Roberts (1983) 
and later developed and tested by Centeno et al. (2006, 2009) and Khomenko et al. (2008), in which changes in the radiative relaxation time associated with small scale magnetic structures are responsible for increasing the cutoff period. This mechanism has also been demonstrated to enable propagation of 5-minute oscillations, even in vertical magnetic structures. However, the underlying basis of this theory and the related simulations is a highly simplified energy equation in which radiative losses are approximated by Newton's law of cooling (Khomenko et al. 2008 and references therein).

A related subject that has been studied extensively is the generation and propagation of chromospheric jets such as spicules (type I and II), macrospicules, surges, fibrils, and mottles. A variety of models have been proposed over the years (see Sterling 2000 for a review), and although it remains unclear whether the many types of jets are different manifestations of the same underlying physical phenomenon or not, there are at least strong indications that the jets known as dynamic fibrils are driven by shock waves traveling through the chromosphere (Hansteen et al. 2006; De Pontieu et al. 2007; Heggland et al. 2007; Martínez-Sykora et al. 2009). Many of these jets have lifetimes around 5 minutes, and so the waves driving them need to have been channeled into the upper chromosphere via one of the processes outlined above.

In this paper, we present the results of two-dimensional simulations of wave propagation from the convection zone to the transition region and corona. The simulations include a sophisticated treatment of radiative losses and heat conduction, and study the effects of different magnetic field geometries and strengths on the propagation of waves through the chromosphere. We also look at the jets that these waves produce once they reach the transition region, and perform a statistical comparison of the jets produced in a model with vertical field and in one with inclined field.

In $\S 2$ we describe the different simulations and the code. $\S 3$ contains an analysis of wave propagation and periodicities in the various models, and the results are discussed in $\S 4$. $\S 5$ looks at jet formation and properties, and a summary and conclusions follow in $\S 6$.

\section{SIMULATIONS}

The simulations have been run using the Bifrost code; a detailed code description has recently been published (Gudiksen et al. 2011). It is a 3D MHD code that is designed to model the solar atmosphere to a high degree of realism by including as many of the relevant physical processes as practical and possible. The code is staggered such that scalars are defined on the grid interior while fluxes are defined on the edges between computational cells. Sixth order polynomials are used to calculate derivatives. High order interpolation is also used when a given variable needs to be evaluated half a grid cell away. In order to maintain stability, artificial viscosity and resistivity terms with strengths that can be set by the user are included following a hyperdiffusive scheme, such that regions of large gradients are also those with the largest viscosity and magnetic diffusivity. This allows the diffusivity to be low throughout most of the simulation box, only becoming significant where the conditions require it.
Table 1

Summary of simulation properties

\begin{tabular}{lccl}
\hline \hline Model & Grid cells & Dimensions $[\mathrm{Mm}]$ & \multicolumn{1}{c}{ Magnetic field } \\
\hline Case A & $512 \times 325$ & $16.61 \times 15.80$ & Extremely weak \\
Case B & $512 \times 325$ & $16.61 \times 15.80$ & Moderate, vertical \\
Case C & $400 \times 535$ & $11.17 \times 14.08$ & Strong expanding tube \\
Case D & $512 \times 325$ & $16.61 \times 15.80$ & Moderate, inclined \\
Case E & $512 \times 325$ & $16.61 \times 15.80$ & Strong, inclined \\
\hline
\end{tabular}

The code includes thermal conduction along magnetic field lines, computed implicitly using a multigrid algorithm. A "realistic" equation of state is pre-computed based on LTE, and stored in tabular form to compute temperatures, pressures, and radiation quantities, given the mass density and internal energy. The code solves the equations of radiative transfer for the photosphere and lower chromosphere, including scattering, using the multigroup opacity methods developed by Nordlund (1982) and Skartlien (2000) in short characteristic form (Hayek et al. 2010). In the upper chromosphere, transition region, and corona, we utilize an advanced radiative loss function consisting of several parts. In the corona and transition region, the optically thin approximation is used, taking into account radiation from hydrogen, helium, carbon, oxygen, neon, and iron using atomic collisional excitation rates from the HAODIAPER atom data package (Judge \& Meisner 1994). In the upper chromosphere, most important lines are not in local equilibrium, and excitation rates are precomputed and tabulated from 1D chromospheric models with radiative transfer calculated in detail (e.g., Carlsson \& Stein 1995, 1997). More details on these radiative loss functions as well as on the code itself can be found in Leenaarts et al. (2011) and Gudiksen et al. (2011).

In sum, the code thus includes most physics that are important in the solar atmosphere; the main omission in the present work is time-dependent ionization, which mainly has an effect in the upper chromosphere and transition region, above a height of $\sim 1 \mathrm{Mm}$. See Leenaarts et al. (2007, 2011) for a discussion of these effects.

The horizontal boundary conditions are periodic, while both the vertical boundaries are open. The upper one is based on characteristic extrapolation of the magnetohydrodynamic variables; the lower one allows outflowing material to leave the box, while the entropy of the inflowing material is set. There is no piston or other imposed external driver producing wave motions; all oscillations are produced self-consistently by the turbulent motion in the convection zone.

We will be analyzing five different simulations, using model atmospheres that are about $16 \mathrm{Mm}$ high. They extend from the upper layers of the convection zone at the lower boundary $(z=-1.4 \mathrm{Mm})$, through the photosphere $(z=0 \mathrm{Mm}$ corresponding to the average height where $\tau_{5000}=1$ ), chromosphere, transition region and corona, with the upper boundary at $z=14.4 \mathrm{Mm}$. The simulation boxes are two-dimensional and contain $512 \times 325$ grid cells, using a uniform horizontal spacing of $32.5 \mathrm{~km}$. In the vertical direction, we use a nonuniform spacing which is $28 \mathrm{~km}$ between each cell from the lower boundary to $z=5 \mathrm{Mm}$, increasing gradually 

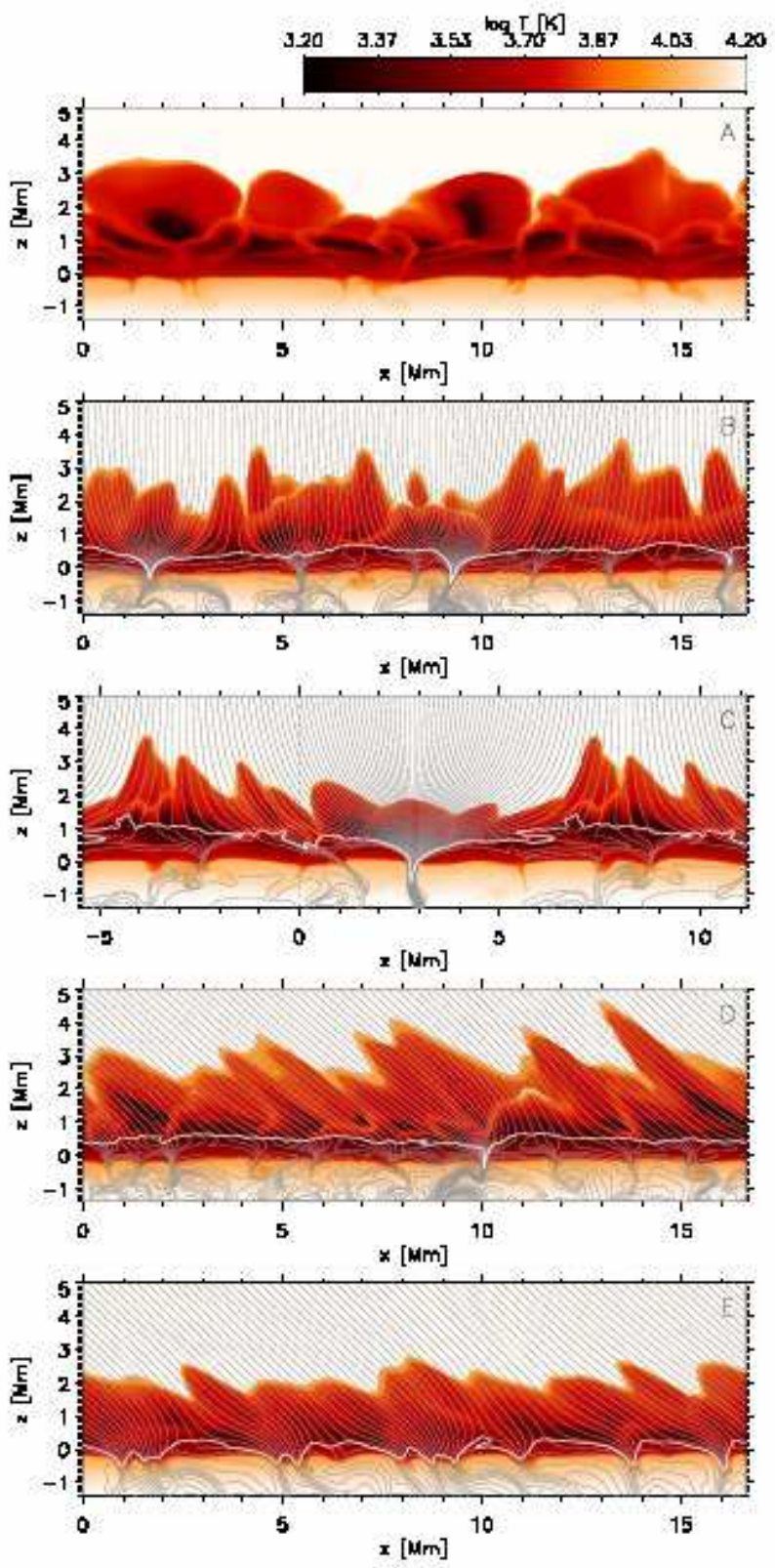

Figure 1. Temperature plot showing the initial states of our five different simulations. The white lines are at the height where the plasma $\beta=1$, while the gray lines are magnetic field lines. Case A is high- $\beta$ throughout and contains only a very weak magnetic field. The dotted vertical black line in case $\mathrm{C}$ marks the location of the horizontal boundary; as this model has a smaller horizontal extent than the others, the rightmost $5.5 \mathrm{Mm}$ have been repeated on the left.

until $z=9.6 \mathrm{Mm}$; from there to the upper boundary it stays at a constant value of $150 \mathrm{~km}$. The properties of our five different simulations are summarized in Table 1. and Figure 1 shows their initial temperature and magnetic field structures. Case $\mathrm{C}$ has slightly smaller dimensions and higher resolution than the others; the other models differ mainly in the strengths and orientations of their magnetic fields. All the simulations have been run for $4500 \mathrm{~s}$, or 75 minutes, of solar time (after an initial relaxation period).
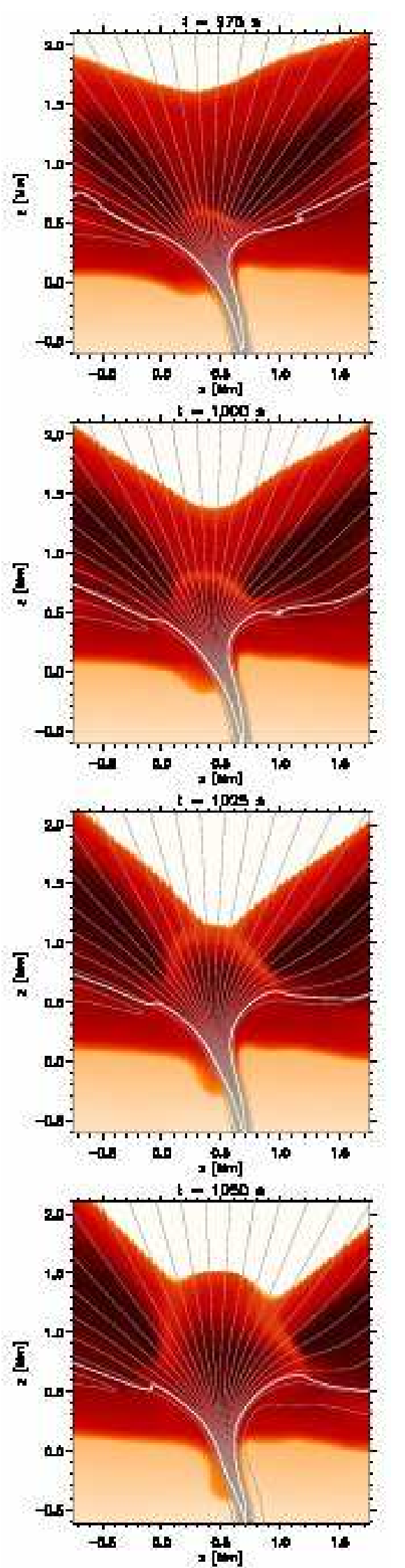

Figure 2. Temperature plot showing a wave propagating upwards and outwards in an expanding flux tube in case $\mathrm{C}$. The white line is at the height where the plasma $\beta=1$, while the gray lines are magnetic field lines. 

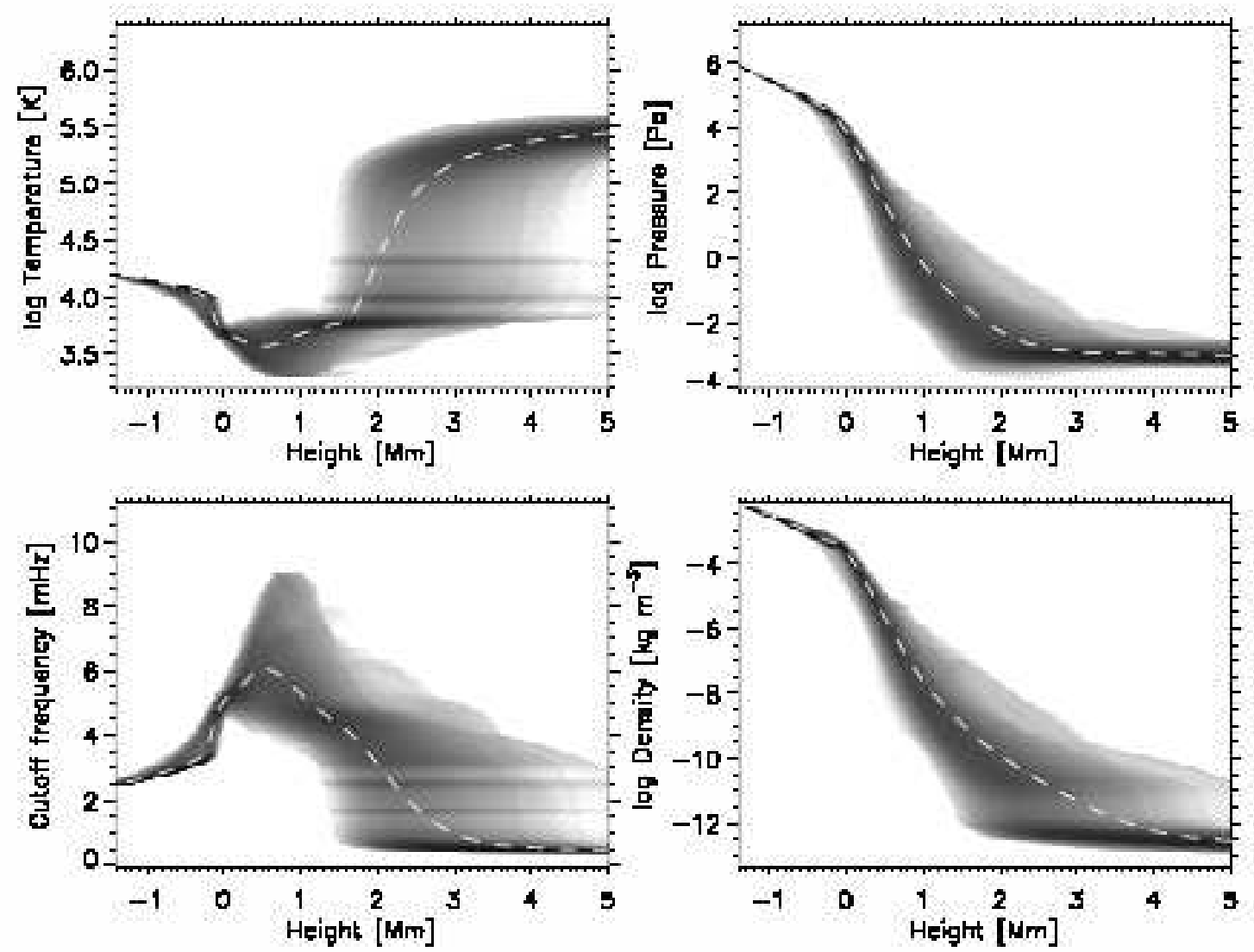

Figure 3. Temperature (top left), cutoff frequency (bottom left), pressure (top right), and density (bottom right) in case D. These are scatter plots showing how often the atmosphere is in a certain state (across all times and horizontal positions); more densely populated states are darker. The white dashed lines are the averages over all times and horizontal positions.

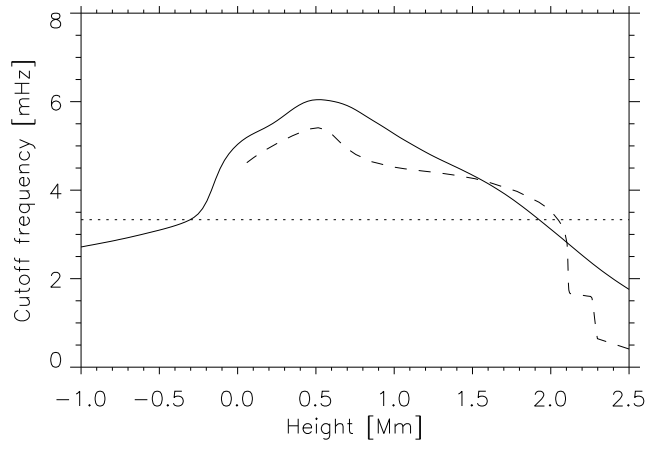

Figure 4. Average cutoff frequency in our case D (solid line) and in the VAL3C solar atmosphere model (dashed line). The horizontal dotted line is at $3.33 \mathrm{mHz}$, corresponding to a period of 5 minutes.

The turbulent motion in the convection zone is able to move magnetic flux tubes around, which means that the field configurations in the high- $\beta$ sections (where $\beta$ is the ratio between the gas and magnetic pressures) of all five simulations are rather dynamic. In contrast, the field in the upper chromosphere and corona in all models except case $\mathrm{A}$ is rather stable and changes little with time. The motion in the convection zone also generates waves that propagate upwards (see Figure 2) and create jets of various lengths and lifetimes once they reach the transition region. Many such jets can be seen in Figure 11. They tend to follow the magnetic field, taking on relatively thin, elongated shapes, but in the non-magnetic case A, they look more like extended spherical waves moving the transition region up and down.

Since we are primarily interested in wave propagation in the chromosphere, not the corona, we omit from our analysis the regions above $z=5-6 \mathrm{Mm}$, the height of the peaks of the tallest jets.

In Figure 3 we show the temperature, pressure, and density structures of case $\mathrm{D}$ in greater detail as functions of height, as well as the cutoff frequency (given by equation 11). The other cases look similar. The temperature plot clearly shows the significant movement the transition region undergoes as jets are continually formed and push it upwards. The three prominent horizontal lines in the temperature structure correspond to the ionization temperatures of hydrogen and helium (the latter twice), and so are an effect of the tabulated equation of state (Leenaarts et al. 2011). The plot of the cutoff frequency shows the region, on average between about $z=0 \mathrm{Mm}$ and $z=1.8 \mathrm{Mm}$ but with large variation in time and space, where the cutoff is above $3.33 \mathrm{mHz}$, corresponding to a period of 5 minutes. This is the region where such long-period waves are evanescent, and which they will have to "tunnel" through if they are to survive into the upper layers of the atmosphere. In Figure 4 we have plotted the average cutoff frequency in our case $\mathrm{D}$ and the cutoff calculated from the semi-empirical VAL3C model (Vernazza et al. 1981). The VAL model 
has a somewhat flatter cutoff in the high chromosphere than our average model, but agrees that the cutoff frequency remains high until one reaches the transition region at about $z=2 \mathrm{Mm}$.

Equation 1, which we used in calculating the cutoff, is strictly speaking only valid in an isothermal atmosphere. There are many possible definitions of the cutoff frequency in a stratified atmosphere (Schmitz \& Fleck 1998; Erdélvi et al. 2007), and the values in Figures 3 and 4 should not be considered exact. The main point is to illustrate that the solar atmosphere contains a height range where the cutoff frequency is higher than the frequencies associated with photospheric oscillations such as $p$-modes.

We should point out that such long-period waves have very long wavelengths, so "local" conditions are not a very well defined term when analyzing them. A wave with a 5-minute period will have a wavelength of 2100$3000 \mathrm{~km}$, given typical sound speeds in the lower atmosphere of $7-10 \mathrm{~km} \mathrm{~s}^{-1}$. The entire height of the photosphere and chromosphere therefore amounts to less than one wavelength. From this, we may expect that even in the absence of any channeling, through field inclination or other processes, a portion of the 5-minute power could make it through to the upper layers. Another expectation is that such channeling does not necessarily have to be active throughout the entire $2 \mathrm{Mm}$ high region, though the effect will likely be greater the more prevalent conditions conducive to channeling are (in both space and time).

This paper aims to bring greater clarity to issues such as the effectiveness of channeling mechanisms and the effects of propagating long-period waves on higher atmospheric layers. To that end, our analysis focuses on two separate but related phenomena: first, the propagation of waves of different periodicities through the atmosphere and the influence upon them by the magnetic field, and second, the jets produced by these waves once they reach the transition region. To study the former, we perform an extensive Fourier and wavelet analysis of the five simulations. For the latter, we individually measure properties such as the lifetimes, lengths and maximum velocities of the jets, and make a statistical comparison between case B (vertical field) and case D (inclined field).

\section{WAVE PROPAGATION}

\subsection{Case $A$}

We start by analyzing case A, a reference model that contains only a very weak magnetic field. It is everywhere weaker than $1 \mathrm{G}$, and the model's behavior is expected to be essentially non-magnetic. For consistency with the other models, however, the magnetic field is included in the analysis below. The initial temperature state is shown in the top panel of Figure 1. This model is meant to be representative of conditions in the internetwork.

The turbulent motion of the convection zone generates waves and flows at many different periods and in many locations. Figure 5 shows a plot of the vertical velocity (grayscale) as a function of horizontal position $(x)$ and time, at a constant height $(z)$ of $1 \mathrm{Mm}$. The overplot-

\footnotetext{
${ }^{2}$ Upward velocity is taken to be positive.
}

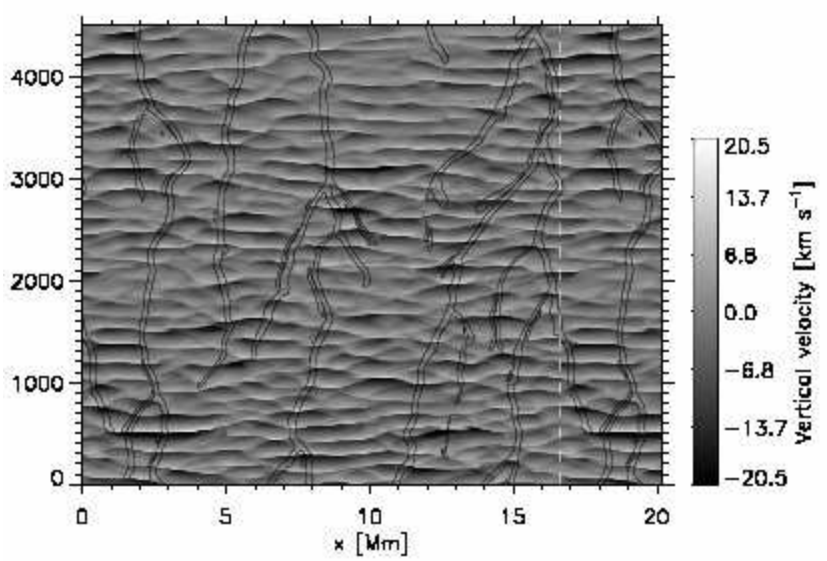

Figure 5. Vertical velocity at $z=1 \mathrm{Mm}$ in case A, with superimposed contours showing the regions where $B_{z}$ at $z=0 \mathrm{Mm}$ is greater than $0.3 \mathrm{G}$. The white dashed line shows the location of the periodic boundary; the leftmost $3.5 \mathrm{Mm}$ are repeated on the right.

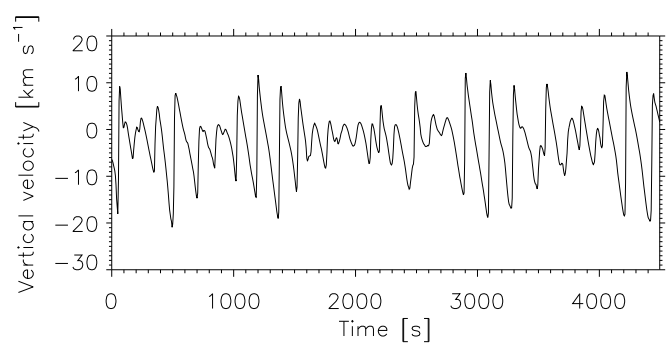

Figure 6. Vertical velocity at $z=1 \mathrm{Mm}$ and $x=1.5 \mathrm{Mm}$ in case A.

ted black contours, for consistency with the other cases, mark the locations where the magnetic field is strongest; specifically, where the vertical component of the magnetic field at $z=0 \mathrm{Mm}(1 \mathrm{Mm}$ below the height at which the velocity is plotted) is $0.3 \mathrm{G}$ or greater. Obviously, these "flux concentrations" are still very weak. Since one of the flux concentrations lies right on the (periodic) boundary, the plot shows the leftmost $3.5 \mathrm{Mm}$ of the box again on the right; the location of the boundary is marked with a vertical white dashed line. In this case, the wavefronts appear to be rather uniformly distributed, with no strong clustering into specific regions either in time or space. They are typically horizontally coherent on length scales of 3-5 Mm.

Figure 6 shows the vertical velocity at the horizontal position $x=1.5 \mathrm{Mm}$, allowing us to see the wave shapes in more detail. The waves are notably asymmetric, showing the characteristic " $\mathrm{N}$ "-shape of compressive waves that have steepened into shocks as a result of the density stratification. The amplitude varies quite a bit with time due to the randomness of the photospheric driver. The strongest shock trains appear around $t=1100 \mathrm{~s}$, $t=2900 \mathrm{~s}$, and towards the end of the simulation. At these times, the shocks reach peak-to-peak amplitudes of up to $30 \mathrm{~km} \mathrm{~s}^{-1}$, generally with stronger downflows than upflows. In fact, the time-averaged velocity at this location is a downflow of about $3 \mathrm{~km} \mathrm{~s}^{-1}$, although this does not imply a net downward mass flux, since the shock waves propagating upward have greater densities. The time between velocity peaks is typically around $200 \mathrm{~s}$. 


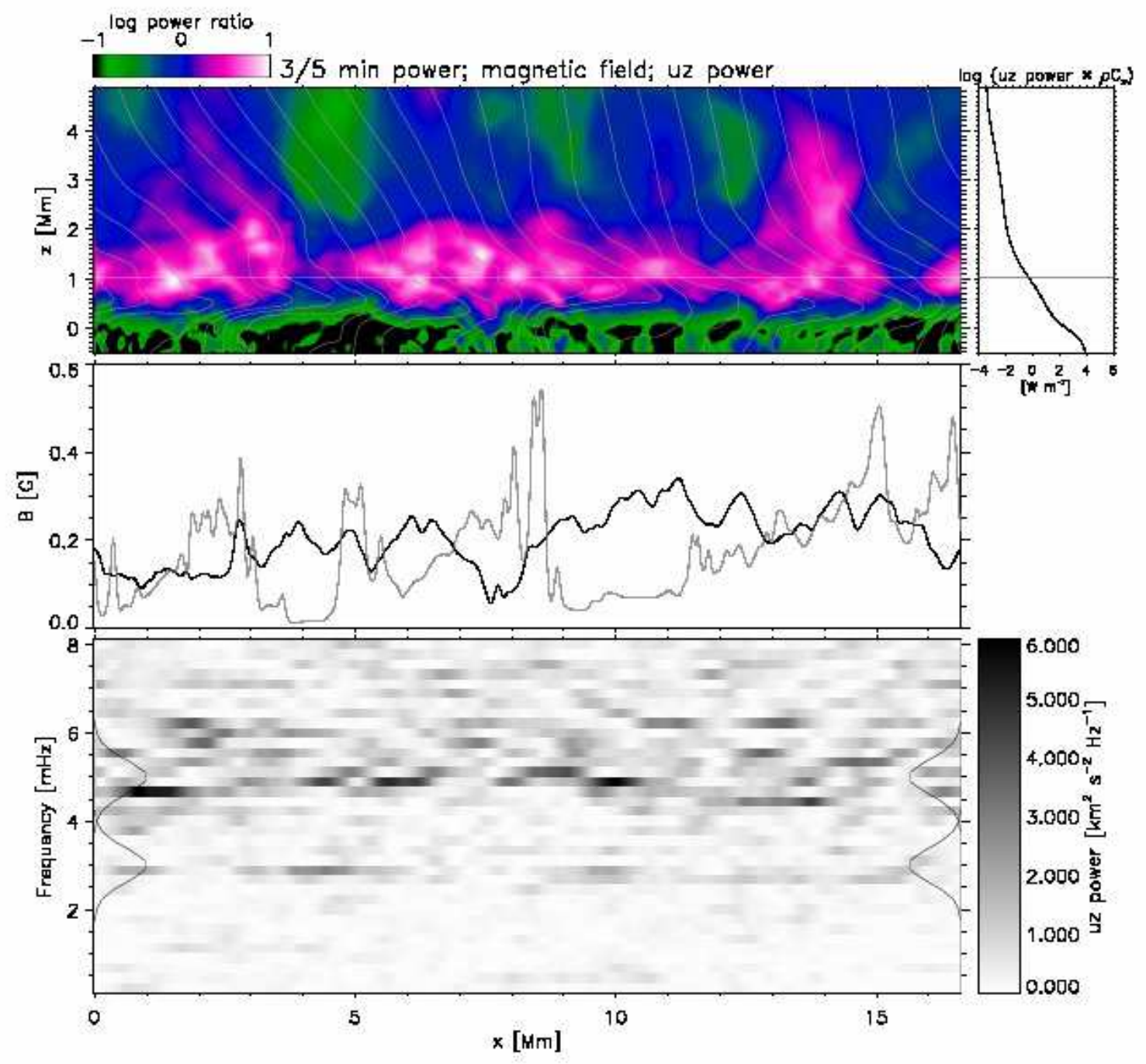

Figure 7. Fourier analysis of case A, showing (top) the ratio of 3-minute power to 5-minute power, with time-averaged magnetic field lines (thin gray lines) superimposed, as well as a horizontal line at $z=1 \mathrm{Mm}$; (top right) the total energy flux as a function of height; (center) the time-averaged magnetic field strength at $z=1 \mathrm{Mm}$ (black) and $z=0 \mathrm{Mm}$ (gray); and (bottom) the power spectrum of the vertical velocity, also showing the Gaussian 3 -minute $(5 \mathrm{mHz})$ and 5 -minute $(3 \mathrm{mHz})$ bands.

We have created power spectra of the simulations by performing Fourier transforms of the velocity at each grid cell. An example of the resulting spectra is shown in Figure 7. The lower panel shows the power spectrum itself as a function of $x$ and frequency $\nu$ at our analysis height of $z=1 \mathrm{Mm}$, the same height where we plotted the velocity field in Figures 5and 6. This height is also marked by the light gray horizontal line in the upper panels. The bell-shaped black curves on either side delineate the two dominant frequency bands used in the subsequent analysis, the "5-minute" band centered at $3 \mathrm{mHz}$ and the "3-minute" band centered at $5 \mathrm{mHz}$. Both are Gaussian bands including contributions at frequencies up to $1 \mathrm{mHz}$ above and below the central frequency. The central frequencies correspond to actual periods of $333 \mathrm{~s}$ and $200 \mathrm{~s}$, respectively.

The upper panel shows the ratio between the power in the 3-minute band and that in the 5-minute band, showing 3-minute dominance as pink and white, 5-minute dominance as green and black, and approximately equal power as blue. Superimposed on this plot are the height where the power spectrum in the lower panel is plotted (light gray horizontal line), and magnetic field lines calculated from the time-averaged field (dark gray). The small panel to the right shows the height profile of the total velocity Fourier power across all frequencies, multiplied by the $x$-averaged density and sound speed to create a measure of the energy flux density rather than the straight velocity power. The middle panel of the figure shows the total time-averaged magnetic field at $z=1 \mathrm{Mm}$ (black) and at $z=0 \mathrm{Mm}$ (gray).

In this model, the 3-minute band is dominant throughout the chromosphere $(z=0.5-1.5 \mathrm{Mm})$. Only in a small area near $x=15.5 \mathrm{Mm}$ does the 5-minute band achieve rough parity, but a look at the spectrum (lower panel) shows that the dominant frequencies at that point are at or above $6 \mathrm{mHz}$, and begin to fall outside the 3 -minute band. In fact, there is significant power at frequencies of $6-7 \mathrm{mHz}$ across most of the simulation box.

This picture, with 3-minute domination throughout, is 


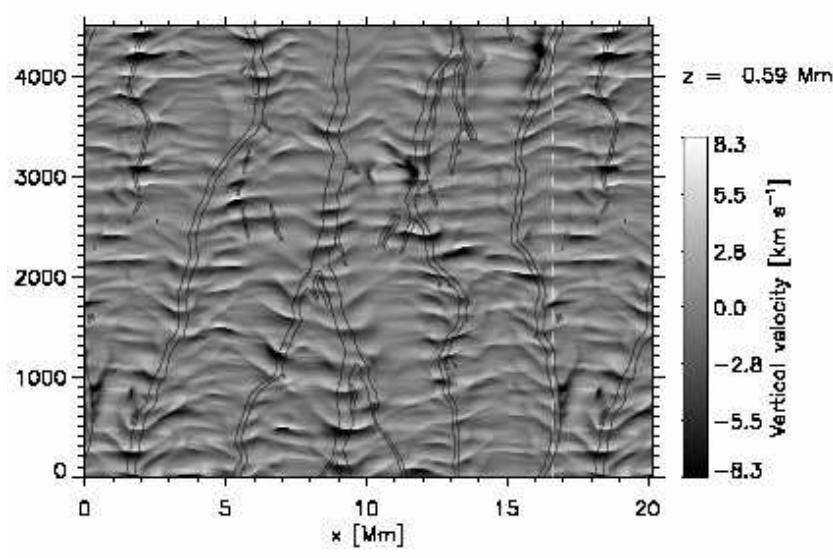

Figure 8. Vertical velocity at $z=0.59 \mathrm{Mm}$ in case B, with superimposed contours showing the regions where $B_{z}$ at $z=0 \mathrm{Mm}$ is greater than $300 \mathrm{G}$. The white dashed line shows the location of the periodic boundary; the leftmost $3.5 \mathrm{Mm}$ are repeated on the right. (An mpeg animation of this figure is available in the online journal.)

typical of the classical results from observations of weakly magnetic regions in the internetwork (e.g., Lites et al. 1993 and references therein). It is a natural consequence of the influence of the acoustic cutoff frequency and the radiative damping of higher-frequency waves. Under chromospheric conditions, the acoustic cutoff frequency is typically around $4.5-5 \mathrm{mHz}$, corresponding to periods of 200-220 s (see Figure 4), and waves at lower frequencies will have difficulty propagating upwards. It is, however, possible to see small amounts of 5-minute power across much of the box, particularly around $x=4 \mathrm{Mm}$, $x=10 \mathrm{Mm}$, and $x=15 \mathrm{Mm}$. This shows that although the long-period waves are damped, their long wavelength still ensures that part of their power reaches $z=1 \mathrm{Mm}$.

\subsection{Case B}

Having studied an essentially non-magnetic model, we now turn our attention to models with magnetic fields of varying strengths and orientations. Case B is a model with several flux concentrations in the photosphere. These flux concentrations are relatively narrow (a few hundred km) and have strengths of 500-1000 G. Such concentrations are a result of the convective motions below the photosphere, which tend to concentrate the magnetic field in relatively narrow regions, often connected with an average downflow, such as intergranular lanes. As we move upwards, the field expands, creating inclined field lines on the edges of the flux concentrations in the chromosphere, while once we reach coronal heights, the field fills all space and the orientation is close to vertical. These effects can be seen in the second panel from the top of Figure 1, which shows the initial state of this case. This model is intended to represent solar conditions in and around network or plage regions.

The velocity field of this case is shown in Figures 8 and 9. As previously, the grayscale image shows the velocity, the black contours outline the field concentrations at $z=0 \mathrm{Mm}$, now using a threshold of $300 \mathrm{G}$, while the white dashed line shows the location of the periodic boundary. Figure 8 shows the vertical velocity at $z=0.59 \mathrm{Mm}$. At photospheric heights, the velocity field is dominated by "global" oscillations with large

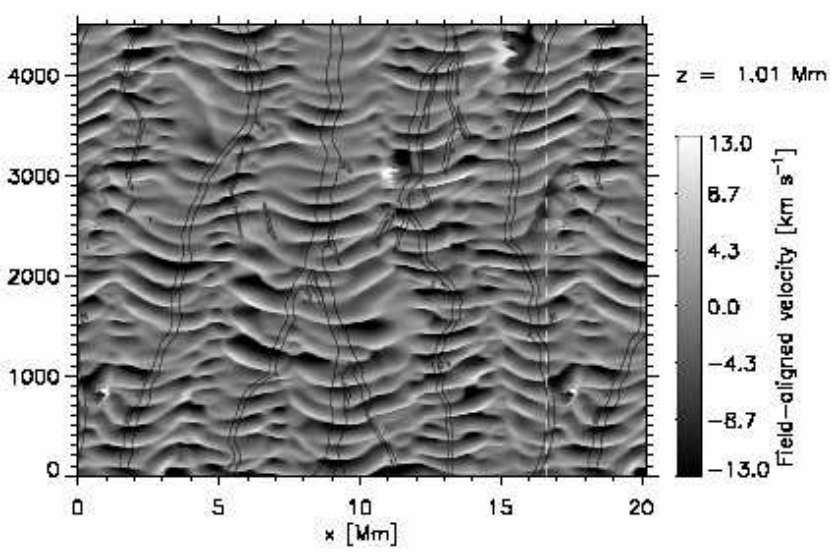

Figure 9. As Figure 8 but showing the field-aligned (rather than vertical) velocity at $z=1 \mathrm{Mm}$. This is the velocity that is used in the subsequent Fourier and wavelet analysis.

horizontal wavelengths and periods around 5 minutes. At $z=0.59 \mathrm{Mm}$, there are still some remnants of these, for example at $x=13-17 \mathrm{Mm}$ after $2500 \mathrm{~s}$, but the shocks generated in and around the flux concentrations are stronger and have begun to fan out. This figure is available as an animation in the online journal, showing how the velocity field develops from $z=0 \mathrm{Mm}$ to $z=2 \mathrm{Mm}$. The animation shows quite clearly how the waves propagate upwards and fan outwards.

Figure 9] shows the velocity along the magnetic field at $z=1 \mathrm{Mm}$, and it is this velocity component we will be performing our analysis on in this and all subsequent cases 3 Since waves are forced to propagate along the magnetic field in regions where the field is dominant, this should give us a cleaner velocity signal than the vertical velocity; the reason the field-aligned velocity was not used in case $\mathrm{A}$ is that it is not a very meaningful quantity in weak-field (high- $\beta$ ) regions.

Unlike in case A, the velocity field at $z=1 \mathrm{Mm}$ is now far from uniformly distributed. As we see, all strong flux concentrations are associated with a region of high velocity amplitude higher up, and nearly all regions of high amplitude are connected with flux concentrations. One main exception is the region of relatively high-frequency signal that starts out at $x=4 \mathrm{Mm}$, eventually moving to $x=6 \mathrm{Mm}$. This area is situated between two flux concentrations and is a region of interference between waves coming from either side - one can see the interference pattern developing in the animated version of Figure 8, which shows the evolution of the wave pattern with height. Some other regions between flux concentrations, for example around $x=11.5 \mathrm{Mm}$ and for the last 1000 s around $x=4 \mathrm{Mm}$, show quite weak signals. The many curved wavefronts are a consequence of the outward propagation of waves from the flux concentrations; particularly notable examples are found at $x=2 \mathrm{Mm}$, $x=6 \mathrm{Mm}$, and near the boundary at $x=16.5 \mathrm{Mm}$. It is also interesting to note how much the flux concentrations are moved around by the convective motion one flux tube starts out at $x=2 \mathrm{Mm}$ and ends up at

3 The sign of the field-aligned velocity denotes whether it is parallel or anti-parallel to the magnetic field. Since all our simulations have unipolar magnetic fields, we take the direction generally corresponding to upward propagation to be positive. 


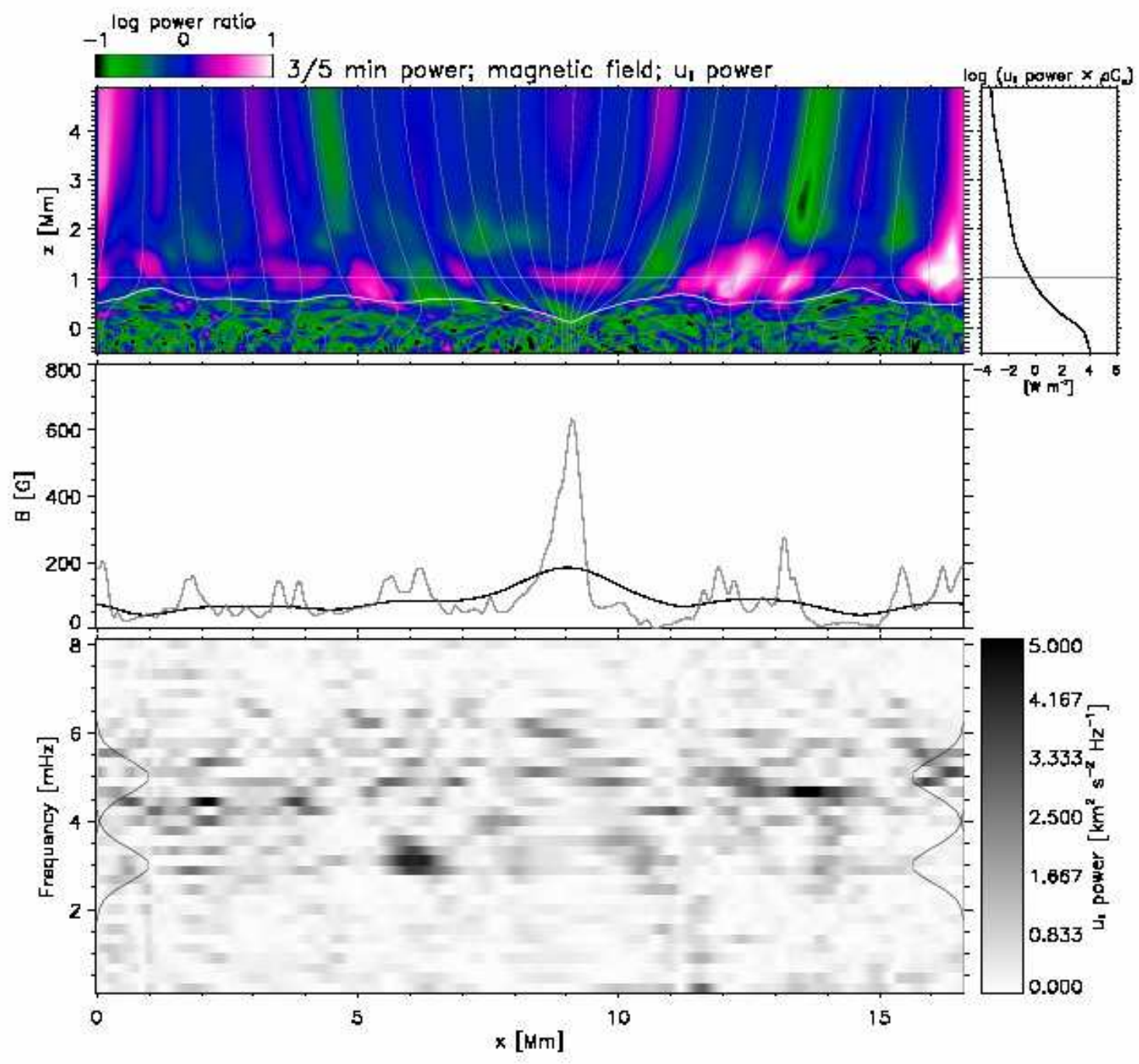

Figure 10. Fourier analysis of case B, showing (top) the ratio of 3 -minute power to 5 -minute power, with time-averaged $\beta=1$ surface (thick white line) and magnetic field lines (thin gray lines) superimposed, as well as a horizontal line at $z=1 \mathrm{Mm}$; (top right) the total energy flux as a function of height; (center) the time-averaged magnetic field strength at $z=1 \mathrm{Mm}$ (black) and $z=0 \mathrm{Mm}$ (gray); and (bottom) the power spectrum of the field-aligned velocity, also showing the Gaussian 3 -minute $(5 \mathrm{mHz})$ and 5 -minute $(3 \mathrm{mHz})$ bands.

$x=6 \mathrm{Mm}$, and several flux concentrations merge during the simulation. This horizontal movement is on the order of $1 \mathrm{~km} \mathrm{~s}^{-1}$, which is within the ranges reported from observations of the movement of bright points in intergranular lanes (Berger \& Title 1996).

As in case A, we have performed a Fourier analysis to investigate the periodicity of the velocity signal. The results are shown in Figure 10, which, like Figure 7 for case A, shows the power spectrum in the bottom panel, the time-averaged magnetic field in the middle panel, and the ratio of power in the 3 -minute and 5 -minute bands in the top panel. As before, the horizontal line in the top panels shows the analysis height $(z=1 \mathrm{Mm})$, and the dark gray lines are time-averaged field lines; the new thick white line indicates the time-averaged height where $\beta=1$, the dividing line between the regions dominated by the magnetic field (above the line) and by gas pressure forces (below it). Although the central flux tube appears very dominant when looking at the time-averaged field strength, it is in fact not that much stronger than the others; however, it moves much less horizontally, making it stronger when averaging over the whole simulation.

The top panel shows that the 3-minute band remains dominant in the chromosphere. This is as expected from classical theory, since the acoustic cutoff period is shortest there, and 5-minute disturbances are expected to be evanescent. It is notable that the peaks in the 3-minute power seem to correspond very well with the peaks in the magnetic field strength. If the effects of the reduced radiative relaxation time in strongly magnetized regions were important, these are locations where 5-minute propagation would be expected.

There are, however, several windows in the chromosphere where there is also significant power in the 5minute band, notably around $x=6 \mathrm{Mm}, x=8 \mathrm{Mm}$, and $x=10.5 \mathrm{Mm}$. The latter two are regions of inclined field on either side of the central strong flux concentration. This fits well with the results of many previous simulations (Suematsu 1990; De Pontieu et al. 2004, 2005; Heggland et al. 2007) that have found that other- 

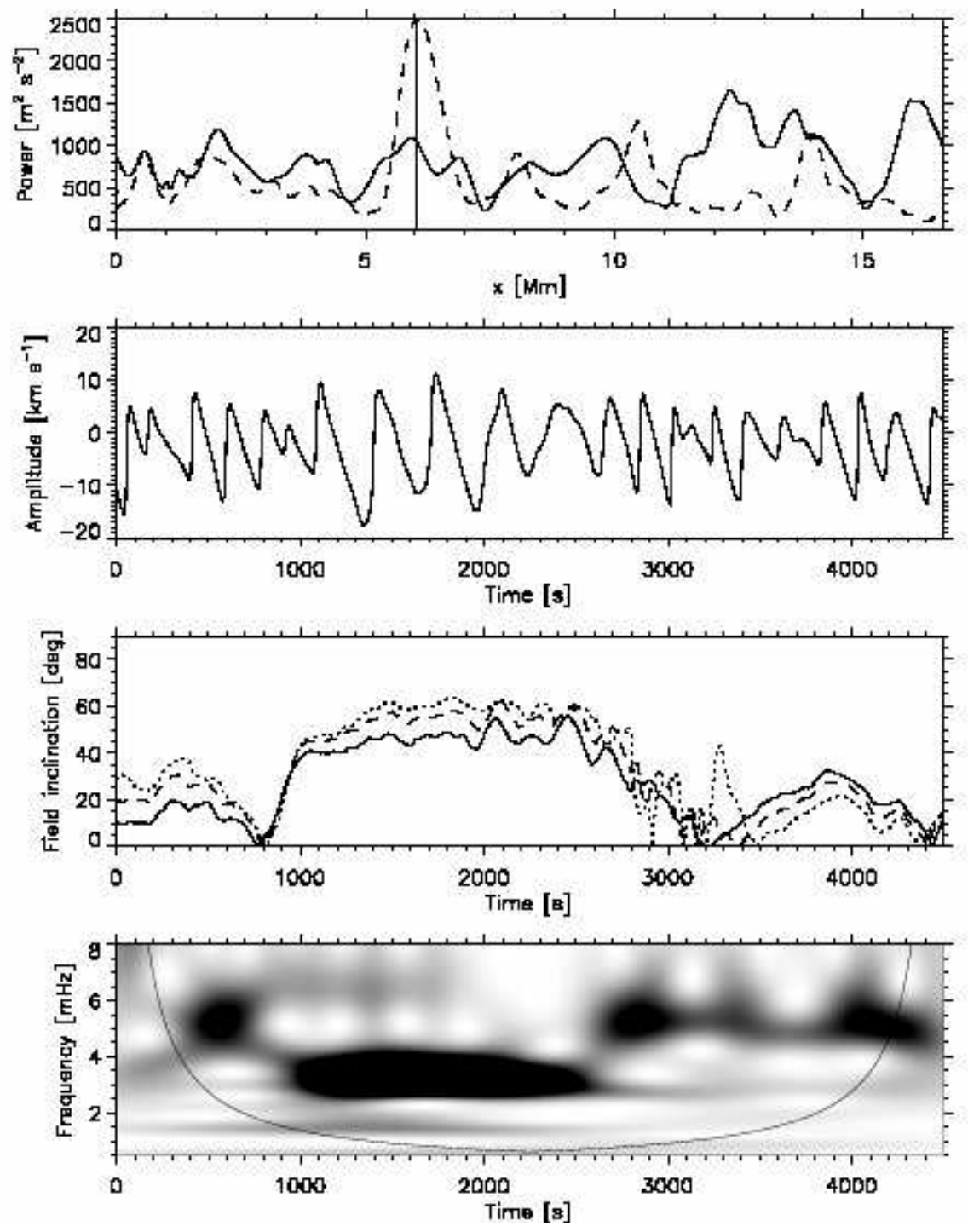

Figure 11. Power (top panel) in the 3-minute (solid line) and 5-minute (dashed line) bands at $z=1 \mathrm{Mm}$ in case B, with a vertical line marking the horizontal location where the other data are plotted; the field-aligned velocity signal (upper middle panel); the magnetic field inclination (lower middle panel; at $z=1 \mathrm{Mm}$, solid; $250 \mathrm{~km}$ below along the field, dashed; $500 \mathrm{~km}$ below along the field, dotted); and the wavelet power spectrum of the velocity (bottom panel). The horizontal position is $x=6.01 \mathrm{Mm}$.

wise evanescent long-period disturbances can still propagate upwards along strong, inclined field, since the reduced effective gravity increases the acoustic cutoff period. We should point out that the previous simulations have generally assumed strong, inclined flux tubes all the way down to the photosphere, while we find that longperiod propagation occurs even when only a part of the propagation is along strong, inclined field at the edges of a mostly vertical flux concentration.

On the other hand, $x=6 \mathrm{Mm}$ is a region where the average field inclination is not particularly high, at least not compared to the regions surrounding it. However, as we have seen from Figure 9, the field is quite dynamic, and the time-averaged field inclination shown in the top panel of Figure 10 does not tell the whole story. In fact, a look at Figure 9 shows that $x=6 \mathrm{Mm}$ connects to two different photospheric flux concentrations over the course of the simulation, and it seems to be particularly during the time when the region is on the outer edge of the central flux tube (from 1000 to $2500 \mathrm{~s}$ ) that it is dominated by longer-period waves. So we need to take the variations with time into account, not just the averages over the whole simulation.

The Fourier analysis deals with averages over the whole analyzed time period, and in order to get sufficient spectral resolution, one typically needs time series on the order of one hour. The solar atmosphere, however, is dynamic on timescales much shorter than that, and when conditions at one location change significantly during the analysis time, the Fourier analysis averages this out and can be misleading. A wavelet analysis, which takes variations in time into account, is a more precise tool; we have performed such an analysis at some selected locations.

In Figure [1] the top panel shows the power in the 3 and 5-minute bands taken from the Fourier analysis, with a vertical line marking the horizontal location, 

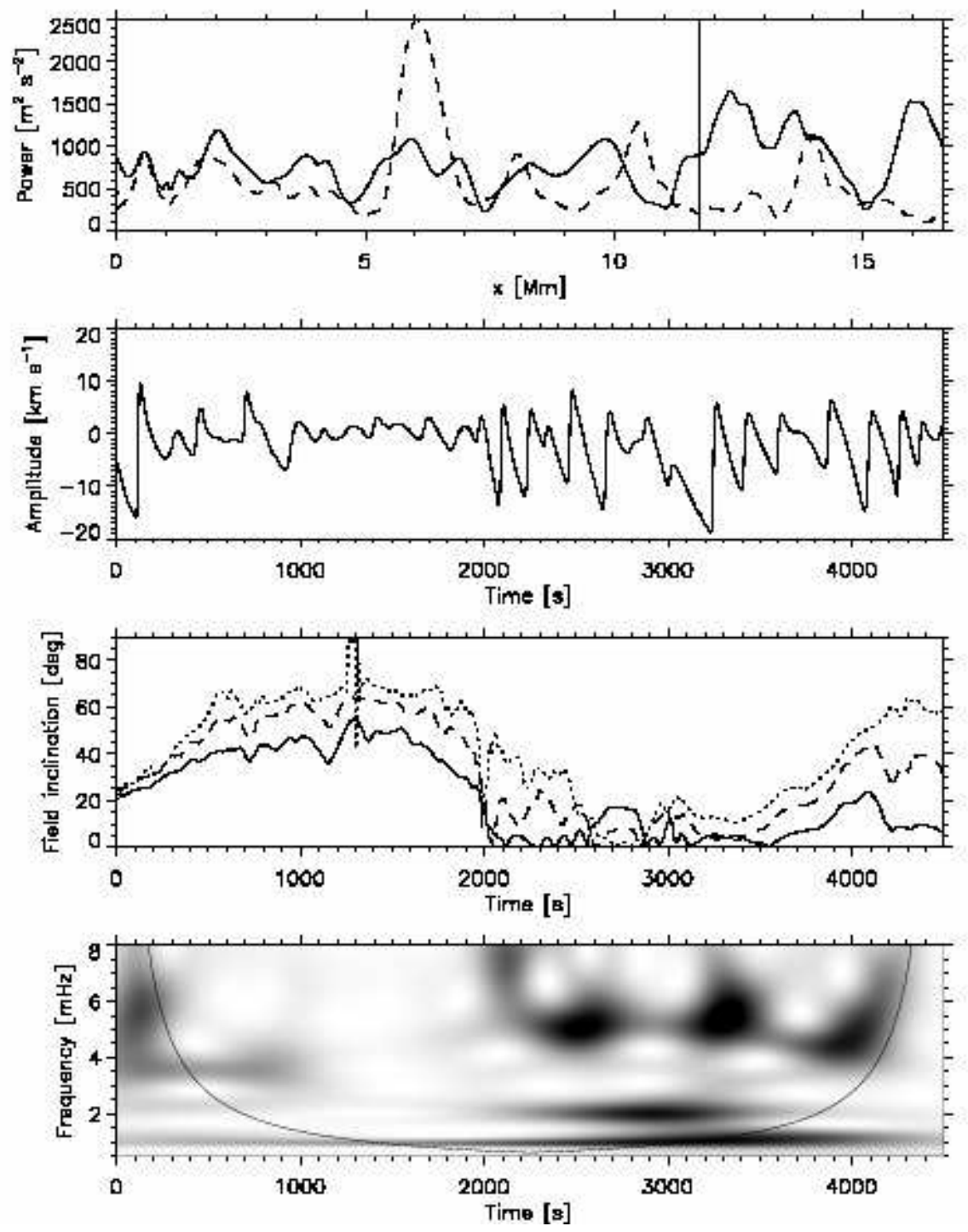

Figure 12. As Figure 11 but at $x=11.70 \mathrm{Mm}$.

$x=6 \mathrm{Mm}$, where we are plotting the data in the three lower panels. The upper middle panel shows the time series of the field-aligned velocity, while the lower middle panel shows the variation of the (absolute) field inclination 4 , both at $z=1 \mathrm{Mm}$. Since the appearance of the wave pattern does not just depend on conditions at this height, but is a function of the conditions throughout the lower atmosphere, we have also traced the field lines downwards at each timestep and plotted, in the same panel, the inclination $250 \mathrm{~km}$ (dashed line) and $500 \mathrm{~km}$ (dotted line) lower along the field. The bottom panel shows the wavelet power spectrum of the velocity signal at $z=1 \mathrm{Mm}$, calculated using the Morlet wavelet (see Torrence \& Compo 1998), with a black line showing the cone of influence; values below this line are subject to edge effects. Darker color corresponds to higher power.

In these plots we see how the period of the velocity signal increases significantly between about 1000 and $2600 \mathrm{~s}$,

\footnotetext{
${ }^{4}$ We use the convention that an inclination of $0^{\circ}$ means the field is vertical.
}

which is the time when this region is located towards the outer edge of the central flux concentration and the inclination is much greater than earlier and later. At this time we get a large peak in the power spectrum at periods around 5 minutes, while at other times, most of the power is in 3-minute waves. This again strongly supports the results in the earlier literature, showing that field inclination is important for long-period wave propagation.

So, do inclination increases always result in increased 5 -minute power? Yes and no. The peaks in the 5-minute power tend to be associated with periods of increased inclination, but the area around $x=6 \mathrm{Mm}$ is unique in having such a strong, relatively pure signal coherent over five wave periods; hence the large peak in the power spectrum there. But even in other locations where the 5 -minute power does not increase markedly, the wave dynamics are still quite different when the inclination is large. An example can be seen in Figure 12, showing the velocity signal and inclination at $x=11.70 \mathrm{Mm}$. Here, the inclination is large for the first $2000 \mathrm{~s}$, and the velocity signal at that time is weaker and more irregular than 
later in the simulation, when the field is more vertical. One explanation for this can be deduced from a comparison with Figure 9. the times when the inclination is large are, in general, the times when the region is located at the interface between two flux concentrations. Since the flux concentrations are the main sources of wave power in the system, such interface regions are also regions of interference between the waves coming from the two flux concentrations. In many cases, this interference is destructive and reduces the amplitude of the disturbances. The periodicity is also perturbed since the signal is now a superposition of the waves coming from the two sources. Examples of regions with such destructive interference are at $x=4 \mathrm{Mm}$ after $3000 \mathrm{~s}$ (a case of triple interference, as there is a weaker, $B_{z}<300 \mathrm{G}$ flux concentration acting as a wave source below this location), and the plotted $x=11.7 \mathrm{Mm}$ for the first $2000 \mathrm{~s}$. In contrast, the regions of most dominant 5-minute power, at $x=6 \mathrm{Mm}$ and $x=10.5 \mathrm{Mm}$, are located towards the sides of the region dominated by the strongest central flux tube, with the interference regions located on the outside of these.

It is also worth noting that the regions of strongest 3minute dominance in the chromosphere are at $x=12-$ $13 \mathrm{Mm}$ and $x=16 \mathrm{Mm}$, both of which are directly above flux concentrations that show relatively little horizontal movement (cf. Figure 9).

\subsection{Case $C$}

While case B had several magnetic flux concentrations of comparable strength, case C contains only one dominant flux tube. Unhindered by the influence of neighboring flux tubes, it can expand freely and fill the entire simulation box at coronal heights. This expansion creates a large region of inclined field at the edges of the flux concentration, which was where we preferentially saw 5minute propagation in case $B$. The initial state of this case is shown in the middle panel of Figure 1.

Although the main flux concentration is strong, with a $\beta=1$ height reaching down to $z=-0.5 \mathrm{Mm}$ (far below the photosphere) at any given time, the magnetic field in the rest of this model is rather weak. As a result, the average height where $\beta=1$ is higher in this case than in case B (see Figure 14); between $x=5 \mathrm{Mm}$ and $x=8 \mathrm{Mm}$, it is above our analysis height of $1 \mathrm{Mm}$, and the field-aligned velocity is not a meaningful quantity there. Because of this, we will be performing the analysis of this case at $z=1.5 \mathrm{Mm}$. Figure 13 (top) shows the field-aligned velocity at that height (grayscale), with superimposed black contours showing where $B_{z}$ at $z=$ $0 \mathrm{Mm}$ is greater than $150 \mathrm{G}$. This value was chosen to point out the secondary flux concentration that merges with the primary at $t=1900 \mathrm{~s}$; the primary has a field strength on the order of $2000 \mathrm{G}$. Since the primary flux concentration is located towards the left of the simulation box, we have plotted the rightmost $3.5 \mathrm{Mm}$ again on the left, with the white dashed line marking the location of the boundary.

Although there are some powerful shocks connected with the secondary flux concentration in the first $1200 \mathrm{~s}$, the velocity field is dominated by the waves propagating outwards from the primary. New oscillations are constantly being generated in the center of the flux concentration. (Figure 2 is an example of such wave propagation, taken from this case; see also Kato et al. 2011 for
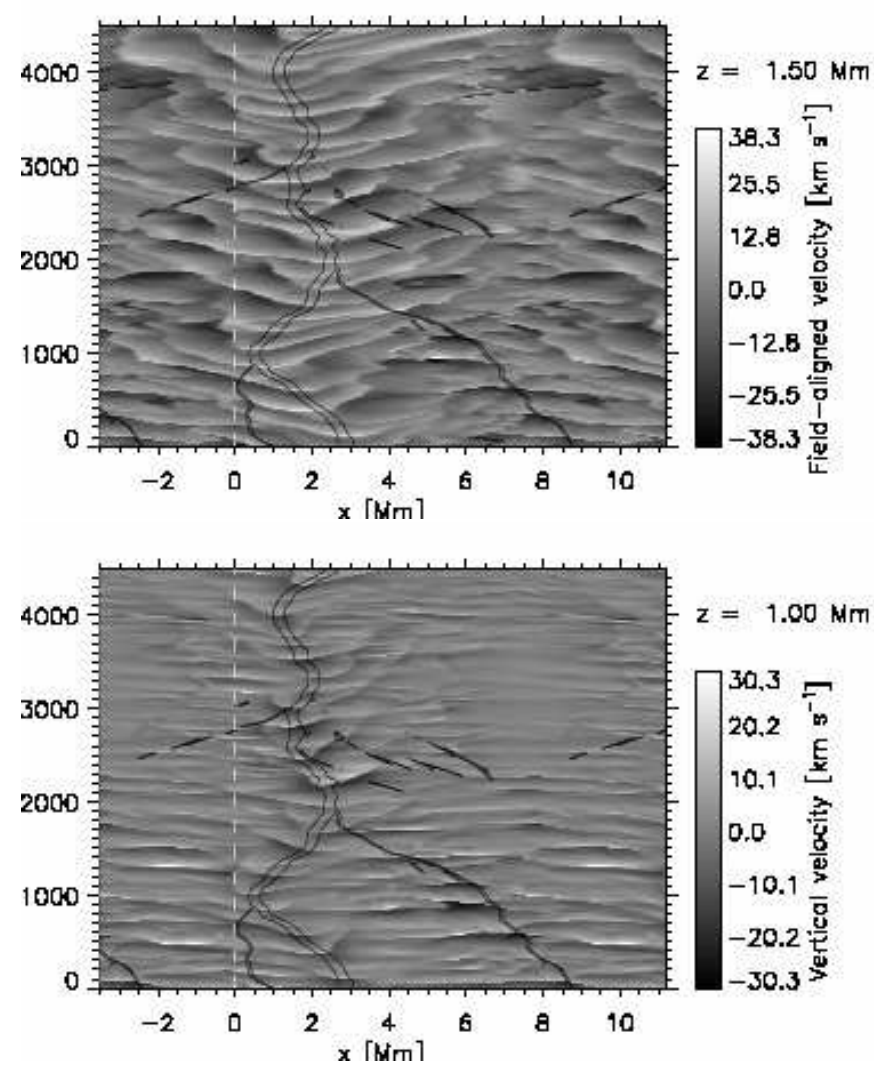

Figure 13. Field-aligned velocity at $z=1.5 \mathrm{Mm}$ (top panel) and vertical velocity at $z=1 \mathrm{Mm}$ (bottom panel) in case C, with superimposed contours showing the regions where $B_{z}$ at $z=0 \mathrm{Mm}$ is greater than $150 \mathrm{G}$. The white dashed line shows the location of the periodic boundary; the rightmost $3.5 \mathrm{Mm}$ are repeated on the left.

a study of wave generation in a similar model.) These propagate upwards and outwards, but there is large variability in how fast they move, and how far to the sides they reach. In general, the waves travel faster and longer in the direction that the flux concentration itself is moving at the time; this is not unreasonable, as the waves from the lower layers will tend to align with the axis of the flux concentration, and this is itself inclined in the direction the flux concentration is moving. We do occasionally see some wavefront merging as a result of these varying propagation patterns, and this could potentially affect the calculated periodicities; however, most of the wavefronts "absorbed" this way are rather weak. Two things are obvious from the picture: although the flux concentration is only a few hundred $\mathrm{km}$ wide at photospheric heights, it dominates a very large area (up to $4 \mathrm{Mm}$ to each side) in the upper chromosphere, and the period of oscillations at its center is significantly shorter than that of the oscillations to the side.

Since the analysis in the other cases is performed at $z=1 \mathrm{Mm}$, we also show the velocity field at that height in this case (Figure 13 bottom panel). As $z=1 \mathrm{Mm}$ is partially below the average $\beta=1$ height, we here plot the vertical velocity rather than the velocity along the magnetic field. In this case, we see wavefronts that are coherent over several Mm horizontal distance, especially in the first $2500 \mathrm{~s}$. These sometimes become very strong shocks 


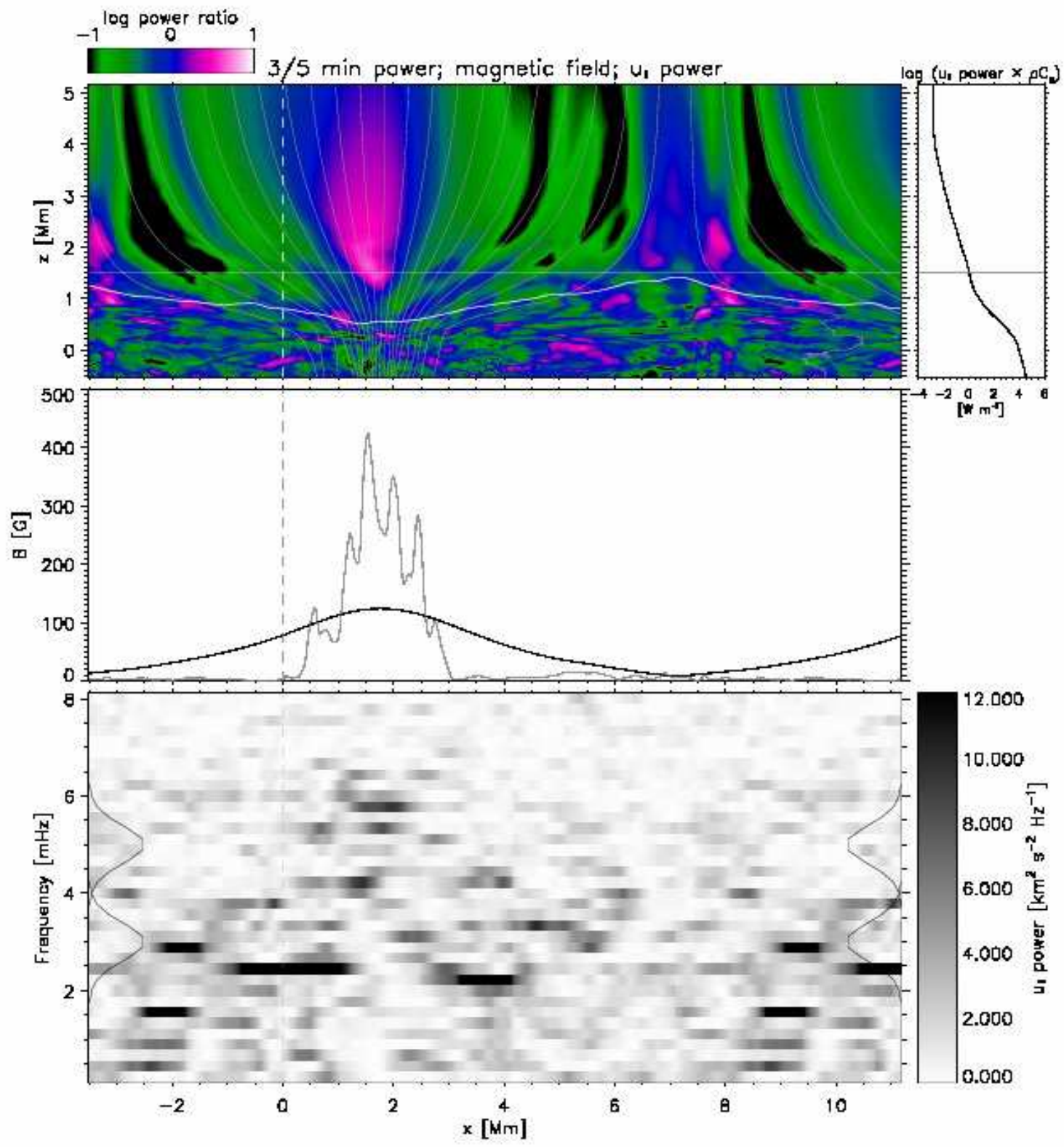

Figure 14. Fourier analysis of case $\mathrm{C}$, showing (top) the ratio of 3 -minute power to 5 -minute power, with time-averaged $\beta=1$ surface (thick white line) and magnetic field lines (thin gray lines) superimposed, as well as a horizontal line at $z=1.5 \mathrm{Mm}$; (top right) the total energy flux as a function of height; (center) the time-averaged magnetic field strength at $z=1.5 \mathrm{Mm}$ (black) and $z=0 \mathrm{Mm}$ (gray); and (bottom) the power spectrum of the field-aligned velocity, also showing the Gaussian 3-minute $(5 \mathrm{mHz})$ and $5-\mathrm{minute}(3 \mathrm{mHz})$ bands. The vertical dashed line shows the location of the horizontal boundary at $x=0 \mathrm{Mm}$.

with amplitudes of more than $30 \mathrm{~km} \mathrm{~s}^{-1}$, and some of the strongest are connected with the secondary flux concentration. The typical period of these oscillations is 200-250 s, though there is some variation. Meanwhile, in part superimposed on the larger "global" pattern, we see the wavefronts clearly propagating outwards from the primary flux concentration. The area farthest from the flux concentration, around $x=8 \mathrm{Mm}$, shows very weak signal in the later part of the simulation. At this time, the magnetic field in the region is close to horizontal, and temporarily lower temperature makes the medium magnetically dominated. This creates a "lid" which inhibits vertical wave propagation.

As in the other cases, we have performed a Fourier analysis, the results of which are shown in Figure 14 A pattern is clear: we see 3 -minute propagation mainly above the center of the flux concentration, and to some extent in the very weak-field area around $x=6-8 \mathrm{Mm}$, and 5-minute propagation in the inclined field regions on the sides of the flux concentration. Although the ratio plot shows this even more clearly at greater heights, it is also plainly visible in the spectrum itself; the central flux tube region between $x=1.5 \mathrm{Mm}$ and $x=2.5 \mathrm{Mm}$ has its whole spectrum shifted towards higher frequencies 


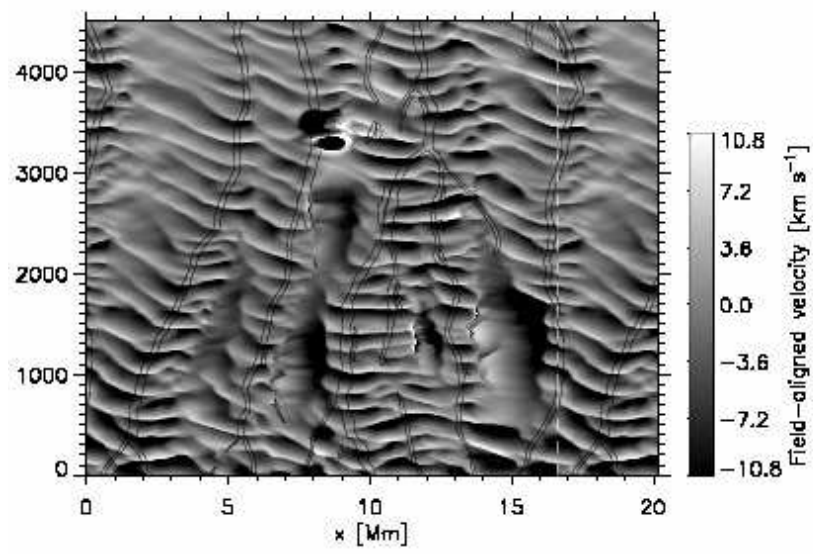

Figure 15. Field-aligned velocity at $z=1 \mathrm{Mm}$ in case D, with superimposed contours showing the regions where $B_{z}$ at $z=0 \mathrm{Mm}$ is greater than $300 \mathrm{G}$. The white dashed line shows the location of the periodic boundary; the leftmost $3.5 \mathrm{Mm}$ are repeated on the right.

than in the regions surrounding it. This is in very good agreement with what we found in case B.

\subsection{Case D}

We now turn our attention to case D, which has similar flux concentrations as case B at the photospheric level, but also includes an additional imposed uniform field of $45^{\circ}$ inclination (slanting towards lower $x$ ). This uniform field is dominant in the corona and upper chromosphere, but is not generally strong enough to dominate dynamics at the photospheric or lower chromospheric levels. The initial state of this model is shown in the second panel from the bottom of Figure 1. This case is meant to represent a plage region with nearby field of opposite polarity.

Figure [15] like Figure 9 for case B, shows the fieldaligned velocity at $z=1 \mathrm{Mm}$ in case $\mathrm{D}$ as a function of $x$ and time, with overplotted black flux concentrations $\left(B_{z}>300 \mathrm{G}\right)$ at $z=0 \mathrm{Mm}$ and the leftmost $3.5 \mathrm{Mm}$ plotted again on the right due to the flux concentration on the boundary. As in case B, we see that the regions with a powerful velocity signal are correlated with the regions of strong flux concentrations below. In fact, the association seems even stronger in case D, with large areas between flux concentrations showing quite weak velocity signals (e.g. between $x=2$ and $x=4 \mathrm{Mm}$ in the later half of the simulation time). Other regions between flux concentrations, e.g. at $x=8 \mathrm{Mm}$ and $x=15 \mathrm{Mm}$, become sites of less regular flows, especially early in the simulation. The field inclination in these regions at those times is generally very large, even near-horizontal in the region around $x=15 \mathrm{Mm}$. The sudden outburst at $x=8.5 \mathrm{Mm}$ between $3200 \mathrm{~s}$ and $3500 \mathrm{~s}$ is due to a reconnection event at $z \approx 0.5 \mathrm{Mm}$. In general, due to the left-leaning field, each flux concentration dominates the velocity in a large area to its left, but only a short distance to its right.

A Fourier analysis of this case is shown in Figure 16 As in case $\mathrm{B}$, the 3 -minute band is dominant in large parts of the chromosphere (notably $x=2-6 \mathrm{Mm}$ and $x=9-14 \mathrm{Mm}$ ), but there are some windows where the 5 -minute band dominates. The most prominent of these are around $x=1 \mathrm{Mm}, x=8 \mathrm{Mm}$, and $x=15 \mathrm{Mm}$. The latter two also exhibit large amounts of low-frequency power, due to the non-periodic flows present there.

Compared to case B (Figure 10), there is less of a connection between the strongest average photospheric fields and the peaks in the 3-minute power, even allowing for the slight leftward drift that would be expected due to the inclination of the additional homogeneous field. This is likely primarily because the flux concentrations in this model display more horizontal movement than in case $\mathrm{B}$ (cf. Figure 16) - for example, the strong flux tube that starts out at $x=10 \mathrm{Mm}$ moves first left to $x=9 \mathrm{Mm}$, and later right to $x=12 \mathrm{Mm}$. Meanwhile, there is a separate flux concentration at $x=13-14 \mathrm{Mm}$ that eventually merges with the other one after $3000 \mathrm{~s}$. The area connected to these is in general the area of greatest velocity power, in particular in the 3 -minute band. It is also the region of lowest average field inclination. The flux concentration at the boundary, $x=16.5 \mathrm{Mm}$, moves comparatively little and this region does correspond to a peak in the 3-minute power. Overall, though, comparison with the average fields and inclinations is of less value in case $\mathrm{D}$ due to the horizontal movement. In order to figure out what is going on, it is necessary to take time variations into account. The wavelet analysis is a tool better suited for this analysis, and we will again look at the time variation of the velocity and inclination at some specific horizontal positions.

Figure 17 is a four-panel figure showing (top) the 3minute (solid) and 5-minute (dashed) power, with a vertical line showing the current horizontal location $(x=$ $0.78 \mathrm{Mm}$ ); (upper middle) the field-aligned velocity as a function of time at that location; (lower middle) the field inclination as a function of time at that location; and (bottom) the wavelet power spectrum. All panels are plotted at the height $z=1 \mathrm{Mm}$; as previously, the field inclination is also shown at two lower heights along the field. The region around $x=0.78 \mathrm{Mm}$ has the highest 5-minute power in the simulation, and it appears to be mainly due to the behavior in the first $1700 \mathrm{~s}$. At that time, there is a quite regular train of long-period shock waves coming up. Between $1700 \mathrm{~s}$ and $3000 \mathrm{~s}$, the velocity signal is much more irregular, correlated with large and rapid variations in the inclination at lower heights, although the dominant periodicity remains 5 minutes. A comparison with Figure [15] shows that the change in behavior happens when the region becomes an interference region between the flux concentration at the boundary and the one starting out at $x=1.5 \mathrm{Mm}$ after roughly $1700 \mathrm{~s}$; before that, it is dominated by waves coming from the latter flux concentration. After about $3300 \mathrm{~s}$, the flux concentration at the boundary has moved to a position just below our observation point; the inclination becomes smaller and we get a wave train with shorter periodicity. The general behavior is similar to what we saw in case B; much of the difference seems to be that with an imposed, left-leaning $45^{\circ}$ field, each flux concentration tends to dominate the wave field in a large region to its left, but only in a small region to its right.

Figure [18 shows the situation at $x=10.99 \mathrm{Mm}$, one of the largest peaks of 3-minute power. Here, the inclination remains mostly below $20^{\circ}$ at $z=1 \mathrm{Mm}$ and $40^{\circ}$ at lower heights, and we get a fairly regular short-period velocity signal, though with varying amplitude. In the last $1000 \mathrm{~s}$, the inclination increases, and this does lead to a more irregular signal, with longer time between the 


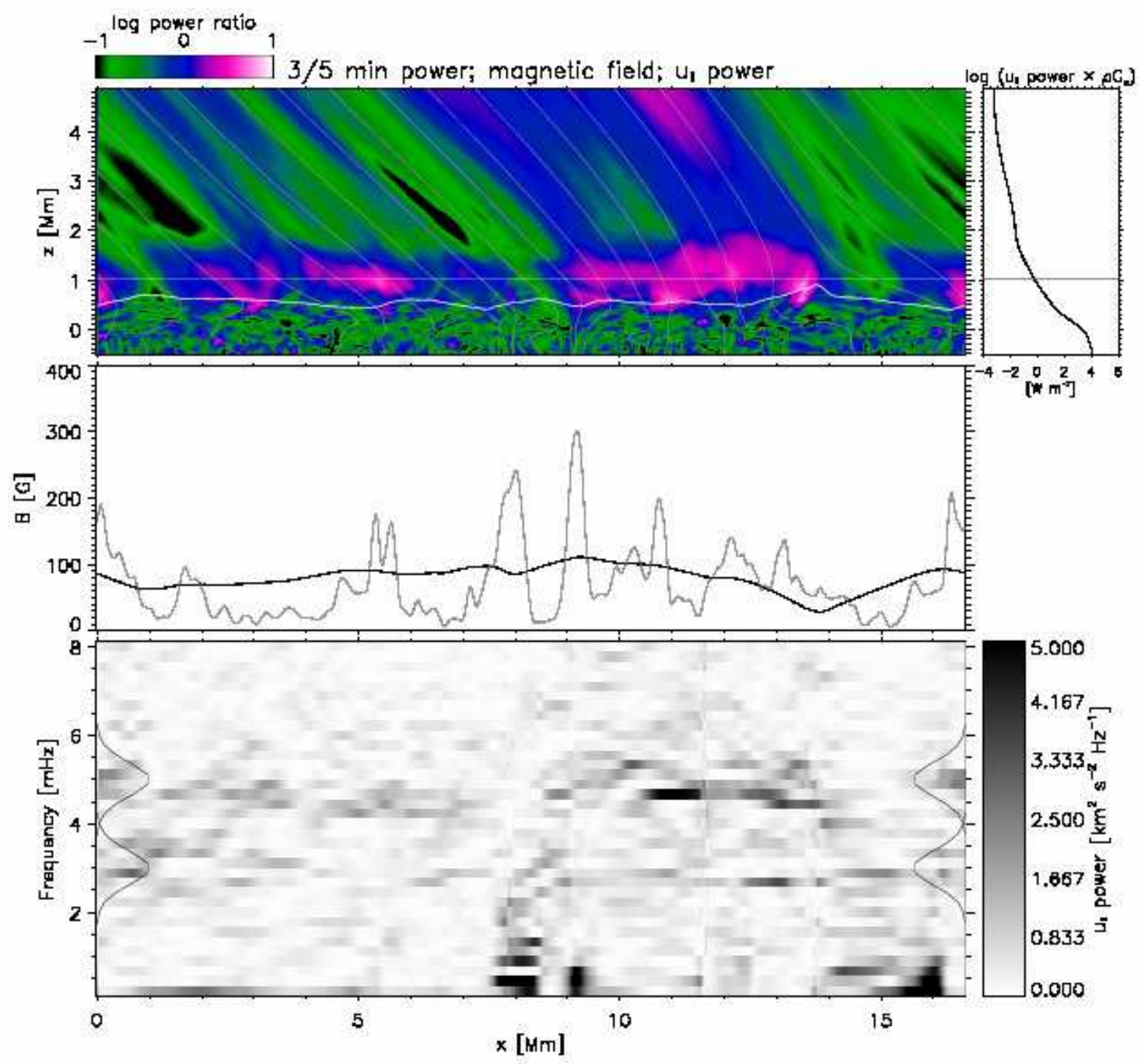

Figure 16. Fourier analysis of case D, showing (top) the ratio of 3-minute power to 5 -minute power, with time-averaged $\beta=1$ surface (thick white line) and magnetic field lines (thin gray lines) superimposed, as well as a horizontal line at $z=1 \mathrm{Mm}$; (top right) the total energy flux as a function of height; (center) the time-averaged magnetic field strength at $z=1 \mathrm{Mm}$ (black) and $z=0 \mathrm{Mm}$ (gray); and (bottom) the power spectrum of the field-aligned velocity, also showing the Gaussian $3-$ minute $(5 \mathrm{mHz})$ and $5-\mathrm{minute}(3 \mathrm{mHz})$ bands.

peaks, though the effect seems noticeable only during the relatively short time when the inclination is above $40^{\circ}$.

Finally, Figure [19] shows the situation at $x=$ 14.82 Mm, at the center of a wide region where the 3minute power is low and the 5 -minute power is dominant. This is a region where the field inclination is generally very large - during most of the simulation, it is more than $60^{\circ}$. A few initial shocks, which actually dominate the power spectrum, are followed by a long period with near horizontal field and a steadily increasing downflow, culminating at $1800 \mathrm{~s}$. Then, after $2200 \mathrm{~s}$, a rather irregular, mostly long-period signal sets in. A comparison with Figure 16 shows that this signal comes from the flux tube on the boundary to the right. A new flux concentration appears (at $z=0 \mathrm{Mm}$ ) after around $2000 \mathrm{~s}$, but it connects only to the left and does not dominate the velocity field directly above it. The most notable effect at this location is the near complete absence of 3-minute signal due to the large field inclination.

In sum, this case shows a behavior that is rather similar to case B, which has a more vertical field. We see 3minute signal above the strongest flux concentrations, where the vertical component of the field is usually strong enough to keep the inclination low, and 5-minute signal towards the edges of the regions dominated by each flux concentration. Among the differences between case B and case $\mathrm{D}$, we find that case $\mathrm{D}$ has less power at high frequencies, connected with interference regions in case $\mathrm{B}$, and that the 3 -minute signal is very weak in regions of heavily inclined field, which were absent in case B. The following case looks into what happens when the strength of the constant inclined field is increased, allowing it to dominate at lower heights.

\subsection{Case E}

In case $\mathrm{E}$, the imposed field is still at a $45^{\circ}$ angle with respect to the vertical, but the field is significantly stronger than in case $\mathrm{D}$. In the mid-chromosphere, around $z=1 \mathrm{Mm}$, it is about 8 times stronger; lower down it is more variable. The field-aligned velocity at 

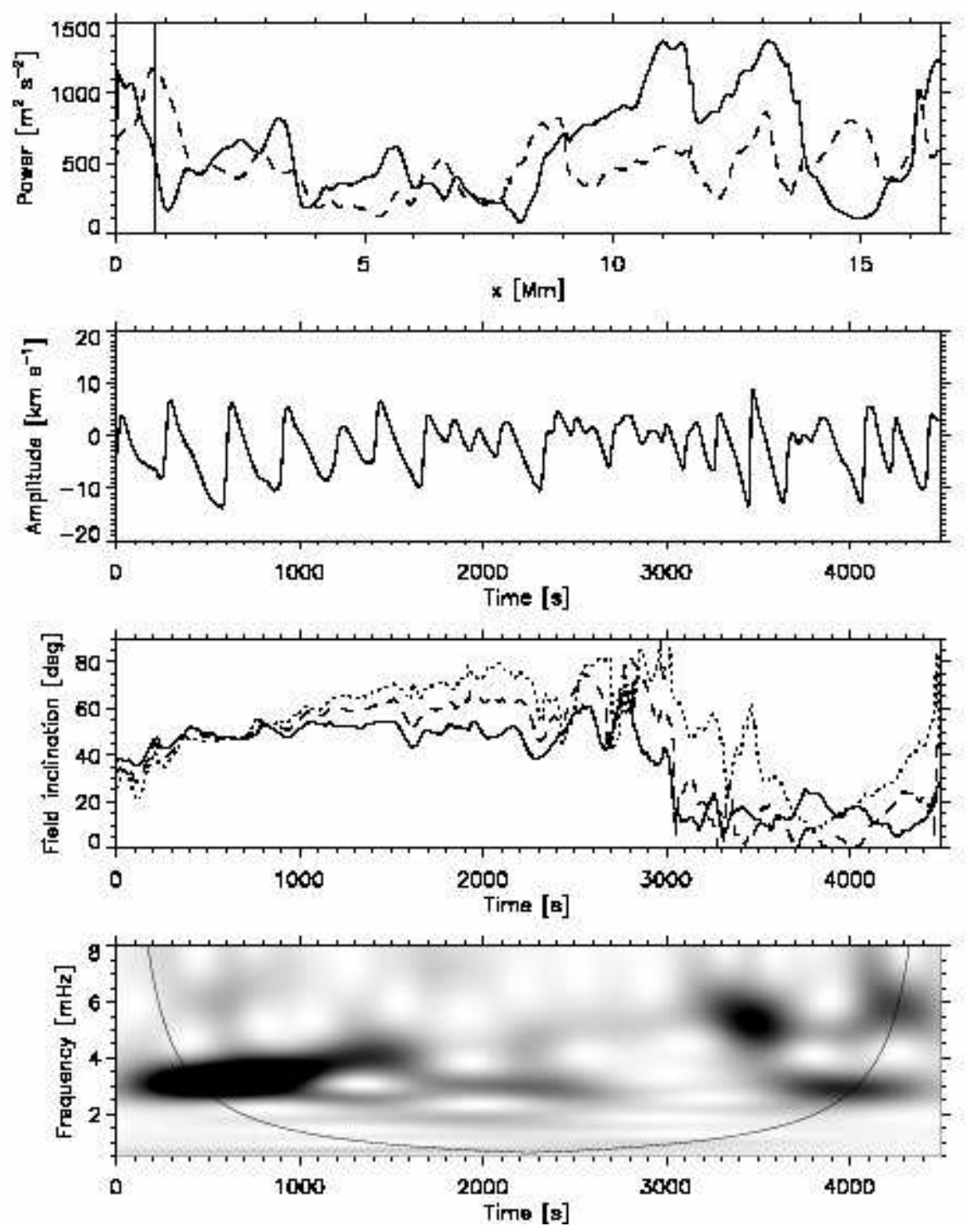

Figure 17. Power (top panel) in the 3-minute (solid line) and 5-minute (dashed line) bands at $z=1 \mathrm{Mm}$ in case $\mathrm{D}$, with a vertical line marking the horizontal location where the other data are plotted; the field-aligned velocity signal (upper middle panel); the magnetic field inclination (lower middle panel; at $z=1 \mathrm{Mm}$, solid; $250 \mathrm{~km}$ lower along the field, dashed; $500 \mathrm{~km}$ below along the field, dotted); and the wavelet power spectrum of the velocity (bottom panel). The horizontal position is $x=0.78 \mathrm{Mm}$.

$z=1 \mathrm{Mm}$ is plotted in Figure 20, with overplotted contours outlining the regions where $B_{z}$ at $z=0 \mathrm{Mm}$ is greater than $1000 \mathrm{G}$ (as compared to the $300 \mathrm{G}$ used in the similar plot for case D, Figure 15). The initial state of the model is shown in the bottom panel of Figure 1 This model represents conditions in very strong plage with nearby field of opposite polarity.

As in the other cases, the regions of highest velocity amplitude seem to be located directly above the strongest flux concentrations. This may seem counterintuitive at first - with such a strong field acting as a wave guide, we might expect the velocity signal to appear to the side of the locations of the flux concentrations. But we should keep in mind that the flux concentrations themselves are fairly wide. Many have widths on the order of $1 \mathrm{Mm}$, or the same as the height we are observing at, so even though the sideways propagation starts around $z=0.5 \mathrm{Mm}$ (see Figure 21), the waves coming from a flux concentration will still tend to be located above it. As we move higher in the atmosphere, the velocity signal does move sideways along the magnetic field, but this effect is not yet very pronounced at $z=1 \mathrm{Mm}$.

Between the flux concentrations, there are channels where almost no wave signal can be seen. These neatly split the computational box into zones of influence for each flux concentration. The channels are actually downflow regions, with slowly varying velocities of $1.5-$ $2 \mathrm{~km} \mathrm{~s}^{-1}$. A single larger downflow event can be seen between $x=1$ and $x=3 \mathrm{Mm}$ after $4000 \mathrm{~s}$.

Figure 21 shows the Fourier analysis of this case. At this field strength and inclination, the 5-minute band is dominant across most of the chromosphere (top panel). Only in two locations, around $x=2.4 \mathrm{Mm}$ and $x=$ $10 \mathrm{Mm}$, does the 3 -minute band dominate. The 3 -minute power is highest above high- $\beta$ regions, i.e. where the magnetic field is weak. This is the opposite of what was 

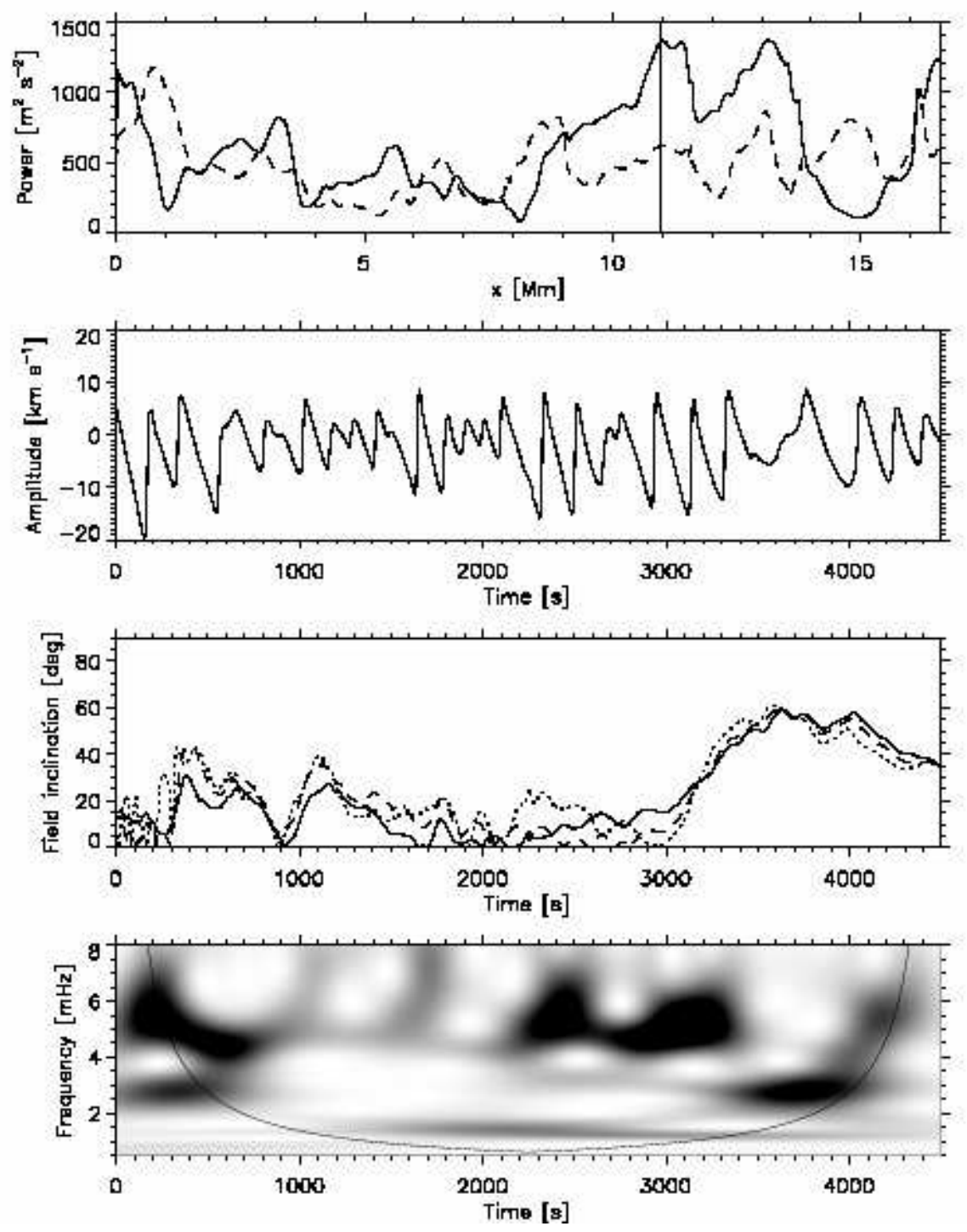

Figure 18. As Figure 17 but at $x=10.99 \mathrm{Mm}$.

found in case B (Figure 10), where the strongest flux concentrations were the sites of highest 3-minute power. In that case, however, the strongest fields were also the most vertical, whereas here, the weaker fields are more vertical than the stronger fields. The results therefore support the idea that the field inclination is central to wave propagation.

The full Fourier spectrum at $z=1 \mathrm{Mm}$ (bottom panel) shows the same situation: significant power in the 5minute band across most locations, though the 3-minute peak at $x=2.4 \mathrm{Mm}$ is the strongest in the simulation. The 5-minute power is more evenly distributed, with one broad peak at $x=11 \mathrm{Mm}$ and some smaller, also quite broad peaks. We also see the very low-frequency power connected with the downflow regions.

Although the strong-field case E has a more steady velocity field than the cases with weaker fields, there is still some drift and variation with time. We once again perform a wavelet analysis in order to look more closely at the wave propagation. Figure 22 shows the results at $x=2.37 \mathrm{Mm}$, the largest peak of 3-minute power.
The velocity amplitude (upper middle panel) is large in the initial stages, when the inclination is relatively low (less than or around $30^{\circ}$ ). There is power at a rather wide range of frequencies at that time, though by far the strongest signal is found in the 3 -minute band between 4 and $6 \mathrm{mHz}$. After $2000 \mathrm{~s}$, most of the signal dies out, as this vertical location becomes part of a rather quiescent downflow region with velocities of $4-5 \mathrm{~km} \mathrm{~s}^{-1}$. After $4000 \mathrm{~s}$, a single larger downflow event occurs, which shows up as power at very low frequencies (below the line marking the cone of influence).

The largest peak of the 5-minute power is centered at $x=10.86 \mathrm{Mm}$, shown in Figure 23, This is directly above a flux concentration (Figure 20) and there is some periodic velocity signal throughout the simulation, though the amplitude varies with time. Again, it is strongest early on, though the peak is mostly within the region subject to edge effects. Although the inclination is below $40^{\circ}$ in the beginning of the simulation, little coherent signal is visible in the 3 -minute band. The 5 -minute band shows power throughout, with a second peak appearing 

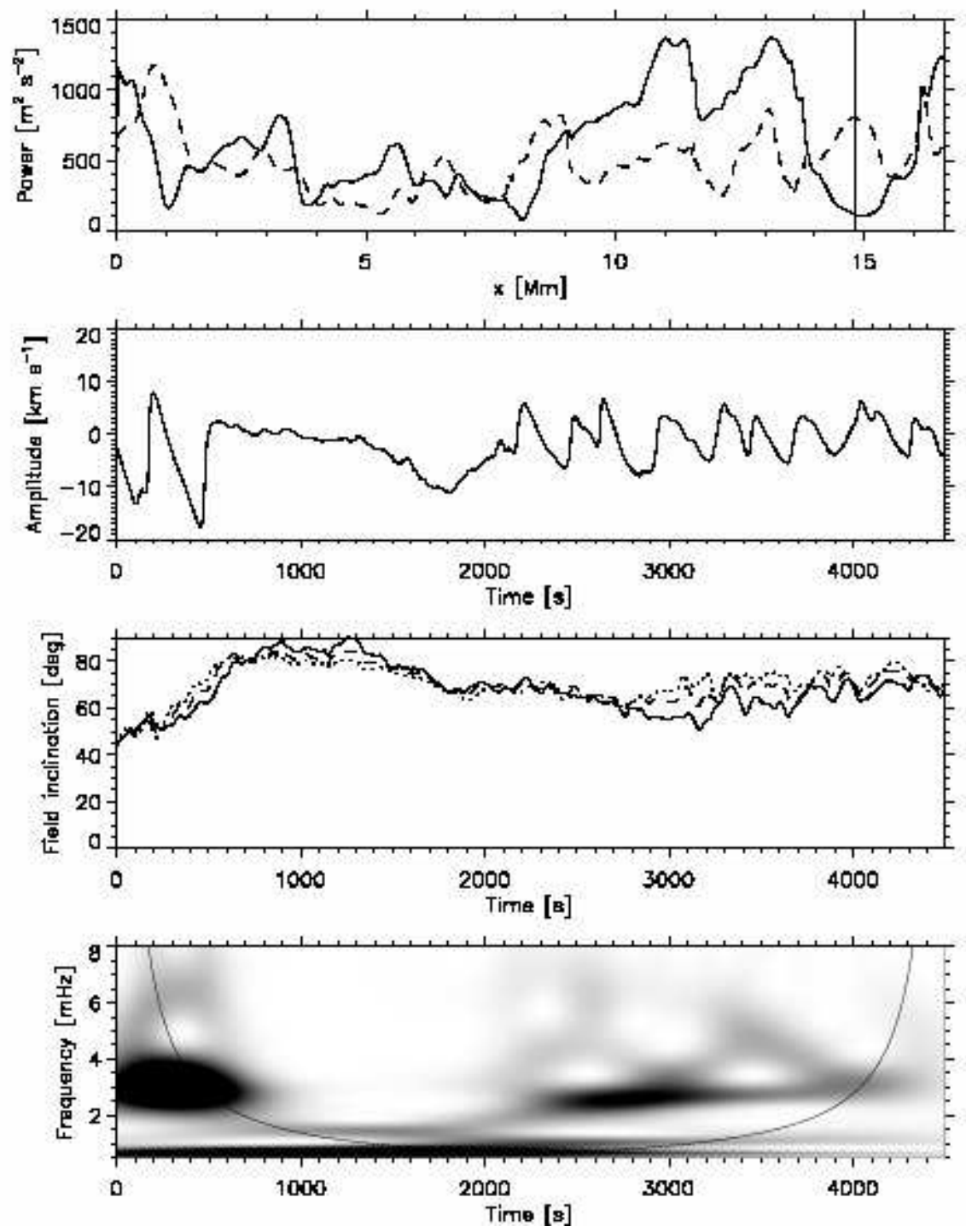

Figure 19. As Figure 17 but at $x=14.82 \mathrm{Mm}$.

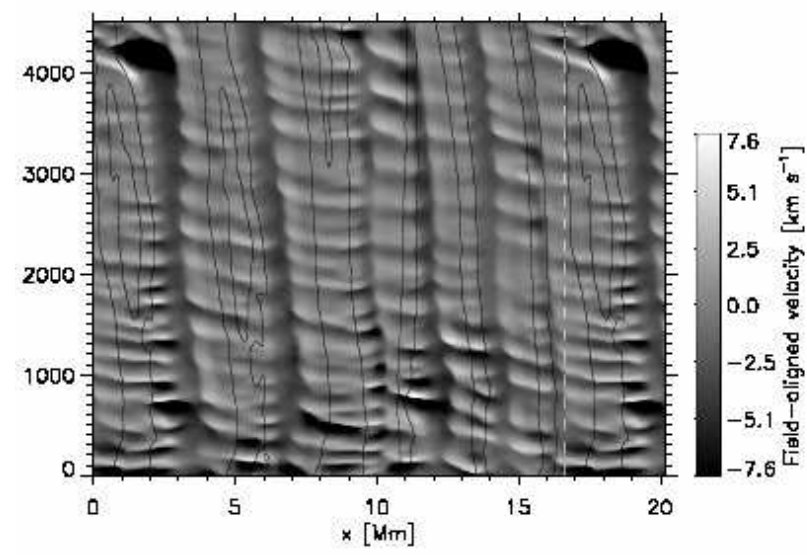

Figure 20. Field-aligned velocity at $z=1 \mathrm{Mm}$ in case E, with superimposed contours outlining the regions where $B_{z}$ at $z=0 \mathrm{Mm}$ is greater than $1000 \mathrm{G}$. The white dashed line shows the location of the periodic boundary; the leftmost $3.5 \mathrm{Mm}$ are repeated on the right. towards the end (again partially within the cone of influence). The field inclination slowly increases with time and ends up at $53^{\circ}$.

\section{DISCUSSION}

The main result from our simulations is that 5-minute waves are able to propagate through the chromosphere in regions where the magnetic field is inclined and sufficiently strong. In regions where the field is vertical or weak, the velocity field is dominated by waves with periods of around 3 minutes.

As mentioned in the introduction, a model which uses the radiative relaxation time, rather than the inclination of the magnetic field, as the mechanism for increasing the cutoff period, has been proposed, and has been demonstrated to work in numerical simulations (Khomenko et al. 2008). However, it relies on a simple Newtonian cooling model for approximating radiative losses.

Our simulations use an advanced and realistic method for computing the radiative losses (Gudiksen et al. 2011) 


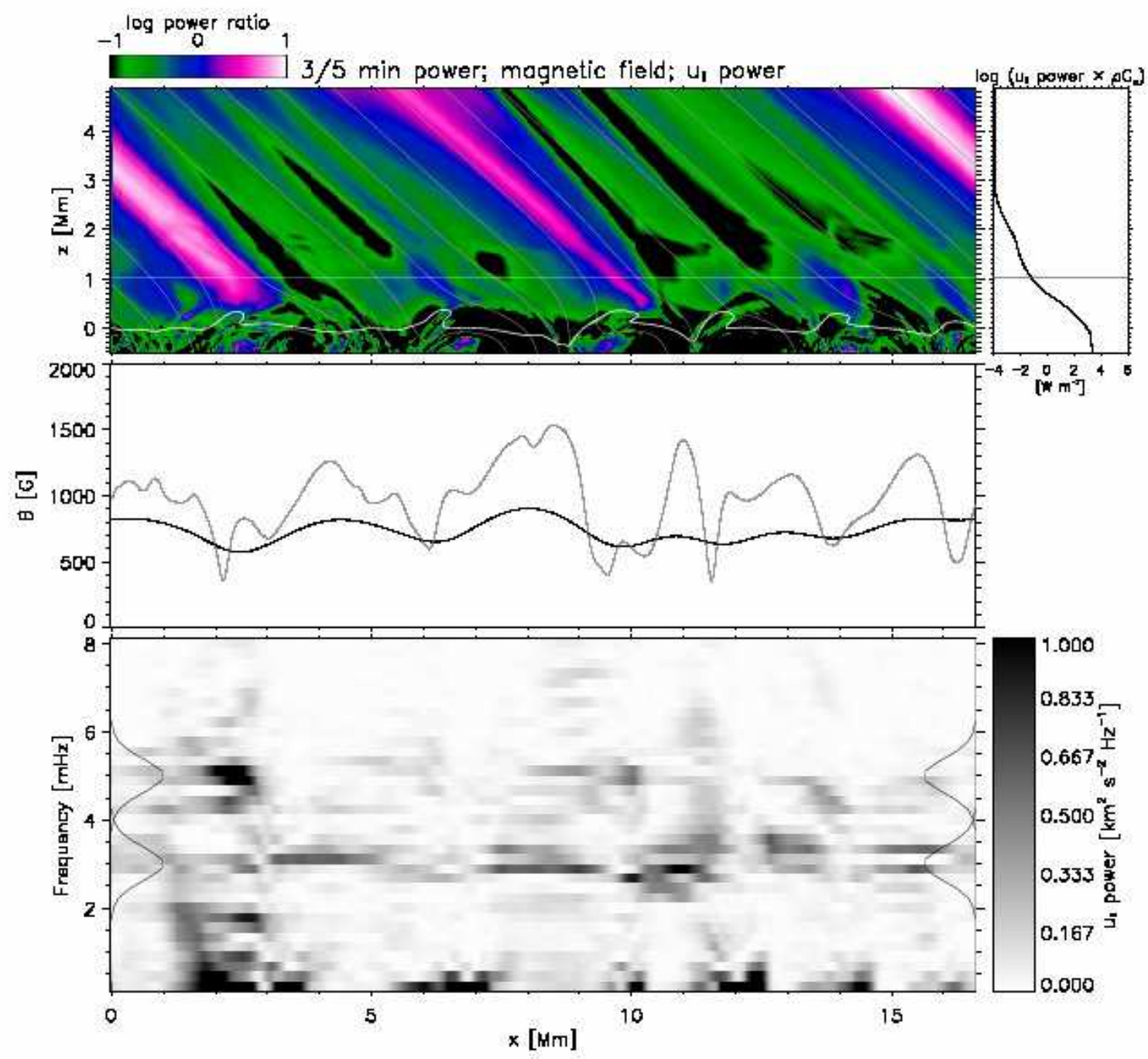

Figure 21. Fourier analysis of case E, showing (top) the ratio of 3 -minute power to 5 -minute power, with time-averaged $\beta=1$ surface (thick white line) and magnetic field lines (thin gray lines) superimposed, as well as a horizontal line at $z=1 \mathrm{Mm}$; (top right) the total energy flux as a function of height; (center) the time-averaged magnetic field strength at $z=1 \mathrm{Mm}$ (black) and $z=0 \mathrm{Mm}$ (gray); and (bottom) the power spectrum of the field-aligned velocity, also showing the Gaussian 3 -minute $(5 \mathrm{mHz})$ and 5 -minute $(3 \mathrm{mHz})$ bands.

that includes all the important mechanisms involved in radiative losses in the photosphere and chromosphere, and as such it is considerably more realistic than a simple Newtonian cooling model. While the radiative relaxation time model expects 5 -minute propagation above all strong small-scale magnetic structures, regardless of the field inclination, our simulations show 3-minute propagation above the central region of flux concentrations, and 5-minute propagation in inclined-field regions to the sides. This is exactly the result predicted by the field inclination model, but is in conflict with the predictions of the radiative relaxation model. Our conclusion, based on the simulations presented here, is therefore that the radiative relaxation model is not effective when a more realistic energy equation is considered.

While large-scale regions of homogeneous inclined field, like our cases D and E, represent idealizations of the conditions at the edge of plages, our case $\mathrm{C}$ represents realistic conditions for an isolated magnetic element or pore. In this model, the magnetic field is largely verti- cal in the photosphere and above the transition region, while the field expansion leads to significant inclination where it is needed the most, in the chromosphere. This can then account for the 5-minute propagation, but we expect 3-minute propagation in the center of the flux concentration. Is this supported by observations?

The answer is in fact not very clear. Lites et al. (1993) show an example of a network region which has very little power at frequencies above $4 \mathrm{mHz}$ over most of its area. There is, however, a pronounced spike of higherfrequency power located in the center of the network $(x=$ $30 \mathrm{Mm}$ in their Figure 4). The authors attribute this to noise from seeing, which it may very well be; it is visible at frequencies up to $20 \mathrm{mHz}$, and a study of the coherence spectra also lends credence to this explanation. However, the signal at frequencies of $5-6 \mathrm{mHz}$ in this spike is much stronger than that at higher frequencies, and indeed is of the same magnitude as the (real) signal at those frequencies in the neighboring quiet regions. It is notable that such a spike, with a spatial extent of 1- 

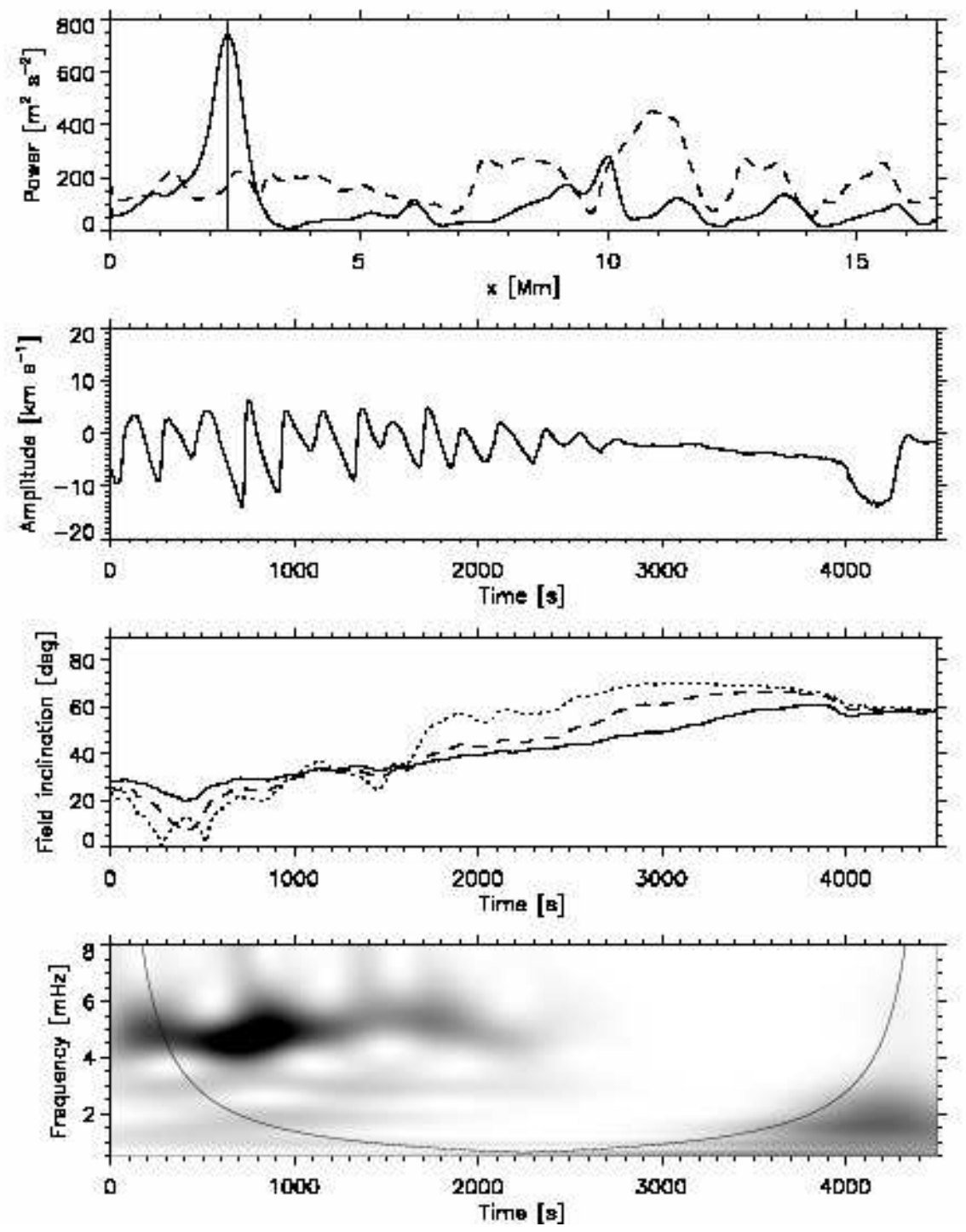

Figure 22. Power (top panel) in the 3-minute (solid line) and 5-minute (dashed line) bands at $z=1 \mathrm{Mm}$ in case E, with a vertical line marking the horizontal location where the other data are plotted; the field-aligned velocity signal (upper middle panel); the magnetic field inclination (lower middle panel; at $z=1 \mathrm{Mm}$, solid; $250 \mathrm{~km}$ below along the field, dashed; $500 \mathrm{~km}$ below along the field, dotted); and the wavelet power spectrum of the velocity (bottom panel). The horizontal position is $x=2.37 \mathrm{Mm}$.

$2 \mathrm{Mm}$, is in fact exactly what we find in our case C. The central spike observed by Lites et al. could thus be real.

In order to make more direct comparisons to their results, which were based on Doppler shifts in observations of the $\mathrm{Ca}$ II H line at $3968.49 \AA$ and the Fe I line at $3966.82 \AA$, we have computed synthetic spectra in these lines from our simulations using the non-LTE radiative transfer code MULTI (Carlsson 1986). The Ca line is treated in full non-LTE, while the Fe line, which is formed in the wing of the Ca line, is included in the same computation using additional localized opacity and source function terms based on LTE. The calculations have been performed column by column, i.e., neglecting any radiative interaction in the $x$-direction. The computed spectra have been smoothed over $2^{\prime \prime}$, which appears to be close to the effective resolution of the observations in Lites et al. (1993), and we have then calculated Doppler shifts from these smoothed spectra.
It should be noted that $\mathrm{Ca}$ II $\mathrm{H}$ has a very complex line profile, usually with several emission peaks within the deeper absorption line. In a dynamic atmosphere, particularly in the presence of strong shocks, these emission peaks can become very large and are often asymmetric (so called bright grains), and the line can undergo central reversal as well. Defining and identifying the line center of such a line is a non-trivial task. We have used a method that finds the center of the region where the intensity is below a certain threshold above the minimum intensity. This method generally gives acceptable results, but can not be expected to correspond directly to the velocity at any one given height in the simulations.

The power spectra of the calculated Doppler velocities are shown in the two right-hand panels of Figure 24. the two left-hand panels show the power spectra of the vertical velocity taken directly from our simulation data, at heights corresponding to the approximate formation 

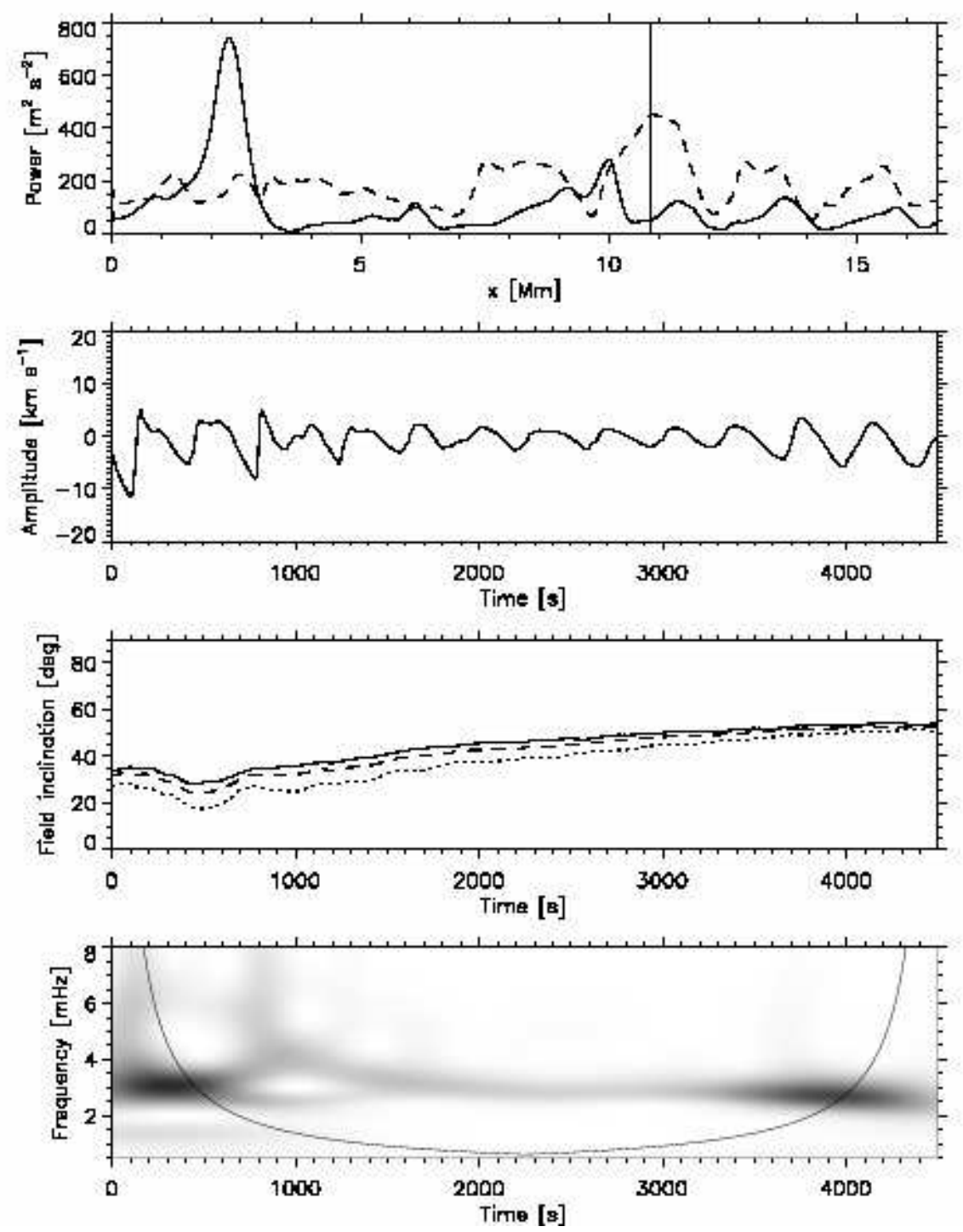

Figure 23. As Figure 22 but at $x=10.86 \mathrm{Mm}$.

heights of the lines. Since the observations of Lites et al. (1993) covered a larger area than our simulation boxes, we show a combination of the spectra from two of our simulations in the figure. In the center of all panels, between $x=16.5 \mathrm{Mm}$ and $x=33 \mathrm{Mm}$, we show the spectrum of our case B, representing network conditions. On both sides, from $x=0 \mathrm{Mm}$ to $x=16.5 \mathrm{Mm}$ and from $x=33 \mathrm{Mm}$ to $x=49.5 \mathrm{Mm}$, we show the spectrum of our case A, representing conditions in the weakly magnetized internetwork. This figure should be compared with Figure 4 in Lites et al. (1993).

While our synthesized Ca power spectrum (upper right) is not a perfect match to the observations of Lites et al., there are many similarities. Of particular note is the difference in the dominant frequencies between the non-magnetic regions on both sides and the network region in the center. The network (case B) is dominated by lower frequencies, particularly in the area between $x=22 \mathrm{Mm}$ and $x=29 \mathrm{Mm}$, where the dominant frequencies are $3-3.5 \mathrm{mHz}$. The internetwork (case A) has a more scattered spectrum, but the dominant fre- quencies are mostly between 5 and $7 \mathrm{mHz}$. Furthermore, the network has significant power at very low frequencies, below $3 \mathrm{mHz}$. These effects are also found by Lites et al.

This power at low frequencies is not found in the simulation velocity at $z=1 \mathrm{Mm}$ (upper left), and although the velocity spectrum and the Ca spectrum are similar in many ways, there are also several differences. These differences are partly due to the smoothing applied to the Ca data, partly due to the difficulty of defining a meaningful Doppler shift of the highly complex Ca line profile, and partly due to the fact that the line is formed over a range of heights rather than at one given height, and this height range can also vary with both horizontal position and time. Using the Ca Doppler velocity as a proxy for atmospheric velocity on the real Sun can therefore be misleading.

The velocity spectrum of the Fe line (lower right) shows a very good correspondence with the velocity at $z=0.25 \mathrm{Mm}$ in the models (lower left). This line has a much simpler profile and is formed in a region with few strong shocks affecting local conditions. The dominant 

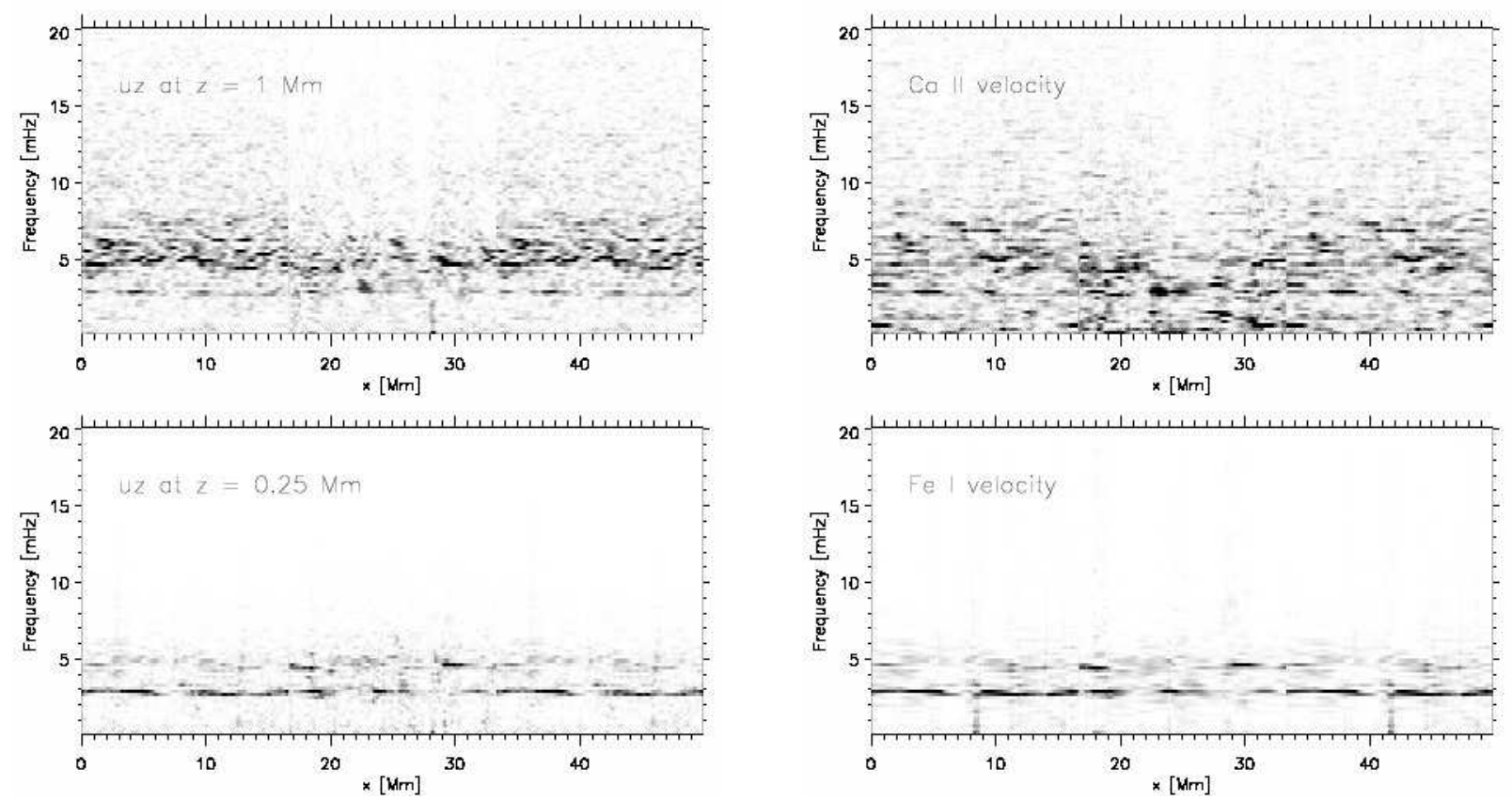

Figure 24. Fourier spectra of the vertical velocity taken from our simulations (left panels) and the Doppler velocity derived from synthesized spectral lines (right panels). The synthesized spectra have been smoothed over 2 arcseconds. In all panels, we show the spectrum of case B in the center (between $x=16.5 \mathrm{Mm}$ and $x=33 \mathrm{Mm}$ ), flanked on both sides by the spectrum of the non-magnetic case A. This figure can be compared with Figure 4 in Lites et al. (1993).

frequency is $3 \mathrm{mHz}$ in both the network and the internetwork, though there is also some power at $5 \mathrm{mHz}$ in most locations. Lites et al. also find that the power in this line is in the $3-5 \mathrm{mHz}$ band, although most of it is between 3 and $4 \mathrm{mHz}$. Like us, they find no significant difference between the spectra in the internetwork and in the network in this line.

More recent observations related to the question of wave periodicity have been performed by Centeno et al. (2009), de Wiin et al. (2009), and Stangalini et al. (2011) (see also Jefferies et al. 2006). Centeno et al. used the Tenerife Infrared Polarimeter of the German Vacuum Tower Telescope at the Observatorio del Teide, with a seeing-limited spatial resolution of $1^{\prime \prime}-1^{\prime \prime} .5$. They then found propagating 5-minute waves in the chromosphere above a facular region. The photospheric magnetic field as determined from Stokes inversions was within $20^{\circ}$ of the vertical. de Wijn et al., using the Solar Optical Telescope on Hinode with a resolution of 0 !' 16 , found 3minute signal in the center of a plage region, but more 5 -minute propagation towards the sides in the direction of the expanding field. Stangalini et al. used a combination of data from Hinode and the IBIS instrument at the Dunn Solar Telescope, with an estimated average resolution of $0{ }^{\prime \prime} 36$. They found propagating 5 -minute waves along the inclined field on the edges of a pore, and some power in 3-minute oscillations at the center. They also found both 5-minute and 3-minute propagation, though with more power in the 5-minute band, in a nearby region with small magnetic elements where they estimate that the chromospheric magnetic field is close to vertical, based on a force-free field extrapolation.

The results of our simulations are in agreement with de Wiin et al. (2009), and with the propagation patterns observed by Stangalini et al. (2011) around their pore. In the more vertical magnetic structures observed by Stangalini et al. (2011) and Centeno et al. (2009), we would expect more power in the 3-minute band than in the 5-minute band based on the results of our simulations, if indeed the field is mainly vertical and the flux tubes do not move around very much. There are, however, several possible mechanisms that could explain the 5 -minute dominance and resolve this apparent difference.

For one, all flux tubes naturally expand with height, and this expansion creates a region of inclined field (as illustrated, on a large scale, by our case C). Thus, even if the field is close to vertical in the photosphere, there will be regions between the photosphere and the chromosphere where the field at the edges of the flux tubes is inclined, and the long-period waves can propagate there. Centeno et al. (2009) do study coherence spectra to look for signs of a possible horizontal shift in the signal as a result of propagation along inclined fields, and find good coherence between the photospheric and chromospheric signal, but our results show that the field does not need to be inclined throughout the photosphere and chromosphere in order to enable 5-minute wave propagation. A few hundred $\mathrm{km}$ along the edges of an expanding flux tube may be enough, and any horizontal shift could then be less than one resolution element $\left(1^{\prime \prime}-1^{\prime \prime} \cdot 5\right.$ in their data). In the observations of Stangalini et al. (2011), the photospheric field is not uniformly vertical, and a forcefree extrapolation is not a very good approximation in the chromosphere. Furthermore, although there is more power in the 5-minute signal, they also find significant signal at periods around 3 minutes in the region with smaller magnetic elements. We believe that our model, where field inclination is the dominant mechanism for allowing long-period wave propagation, is compatible with these findings.

Flux tube movement and limited resolution may also be partially responsible for the relative dominance of 
5 -minute power in the observations of Centeno et al. (2009). In case B, we found that the strongest 3-minute power appeared above flux tubes that undergo little horizontal motion, while 5-minute power was found in inclined field regions at the edges of flux tubes. If the flux tubes move around, both the 5 -minute and 3-minute power will be spread out and one would not see a clear distinction between the (average) flux tube center and the sides in a Fourier analysis. The flux tube center also covers a rather small area at any given time, and this makes the related 3-minute waves difficult to observe in low-resolution data. In the higher-resolution data of Stangalini et al. (2011), regions of 3-minute propagation are found, and this could possibly be because the flux tubes at these locations move around less. We would encourage observers to look for differences in the periodicity of oscillations at the center and edges of flux tubes in future high-resolution datasets.

A third possibility is that heating may play a role. The temperature structure of the chromosphere is in general not well known. In particular, the real Sun may have more magnetic heating of the upper chromosphere than our 2D models. Higher temperature would reduce the cutoff frequency and allow 5-minute waves to propagate more easily.

Yet another suggestion, as mentioned by de Wiin et al. (2009), is that the field may be twisted. The waves could then travel along field lines that are everywhere inclined with respect to the local vertical, but without significant horizontal displacement. Such field twist is a $3 \mathrm{D}$ effect and can not be tested in our 2D simulations, but should be considered in later work.

A different point, that we have already mentioned in our analysis, is also worth making: although a Fourier analysis can be a powerful tool, it has some important limitations. In order to achieve sufficient spectral resolution, one usually needs time series on the order of one hour. The solar atmosphere, however, is dynamic on timescales of minutes. Atmospheric conditions can and do change, and the Fourier transform is not well suited for picking up such changes. At least in areas where the general signal is weak, non-recurring events can end up dominating the power spectrum (see Figure 19 for an example; there are several other examples in the dataset). In such cases, the Fourier analysis does not say all that much about the general conditions at that location. This can be particularly dangerous because the eye is naturally drawn to peaks in the power spectrum. Also, there can be times when local conditions are notably different from the time average, and these can be correlated with changes in the signal (e.g., Figure 11). A wavelet analysis, which takes time variations into account, or at least a careful examination of the time series, is essential for identifying the actual processes going on in the atmosphere.

\section{JET FORMATION}

So far we have been looking at the propagation of waves through the chromosphere. But one of the reasons why we are interested in this propagation is the effect these waves have once they reach the transition region.

Jets of chromospheric material go by many names, such as spicules, mottles, fibrils, straws, macrospicules, or surges. There is no widespread agreement on whether all or some of these represent different aspects of the same underlying phenomenon, or on the driving mechanism or mechanisms behind them. However, there is mounting evidence that at least dynamic fibrils and some mottles appear to be driven by waves coming from below (Hansteen et al. 2006; De Pontieu et al. 2007; Rouppe van der Voort et al. 2007; Heggland et al. 2007). Such wave-driven jets also appear frequently in our simulations here, and we will look into their properties and make a statistical comparison between jets in case B (mostly vertical field) and case D (field inclined $45^{\circ}$ ).

A large number of jets are formed in the simulations, and we have used semi-automatic routines to identify and measure them. We identify the jets as local maxima (in both space and time) of the height where the temperature is $40000 \mathrm{~K}$, corresponding to the lower transition region. We have included snapshots of the tallest jets in the two simulations in Figures 25 (case B) and 26 (case D). We see that they can be quite tall, reaching heights of up to $6 \mathrm{Mm}$, and that their axes follow the magnetic field, as we would expect.

In order to analyze the properties of the jets, we want to look at their time evolution. A rising and falling jet will generally have a parabolic shape in a plot of distance vs. time (e.g., Hansteen et al. 2006), but for the most accurate results, the measurements should be taken close to the central axis of the jet. The jet peaks found previously are used as starting points. In case D, where the magnetic field is inclined, we track the magnetic field line that passes through the jet peak, and measure the variables along this (moving) line through the jet's lifetime. In case B, the magnetic field and the jet axes are generally close to vertical, and we use a simplified procedure taking vertical cuts through the locations of the jet peaks. Experiments using the more involved fieldtracking procedure in case B show that the inaccuracies resulting from the simplified treatment are small.

Using a method similar to the one of Hansteen et al. (2006) and De Pontieu et al. (2007), working on observations, Heggland et al. (2007), working on 1D simulations, and Martínez-Sykora et al. (2009), working on 3D simulations, we produce distance-time diagrams of the temperature and fit the position of the transition region with parabolas. These fits are then used to calculate properties such as the maximum velocity, deceleration, maximum length, and duration of each jet. Jets that do not have a parabolic shape or that represent only minor disturbances are discarded from the analysis.

Note that, as jets sometimes occur in quick succession in the same locations, occasionally they can overlap. This can lead to situations where the descending phase of one jet is cut short by the next jet appearing. This will mainly affect the calculated lifetime of the jet, though it can to a lesser extent also impact the maximum measured length and velocity. The calculated deceleration depends only on the overall shape of the parabola and is largely unaffected. Overall, this does not happen frequently enough to significantly change the statistics, though it can lead to some outliers, particularly with respect to the durations.

In total, we have identified 192 parabolic jets in case B, and 129 in case D. Their properties and various correlations are shown in Figure 27, which is in the same 

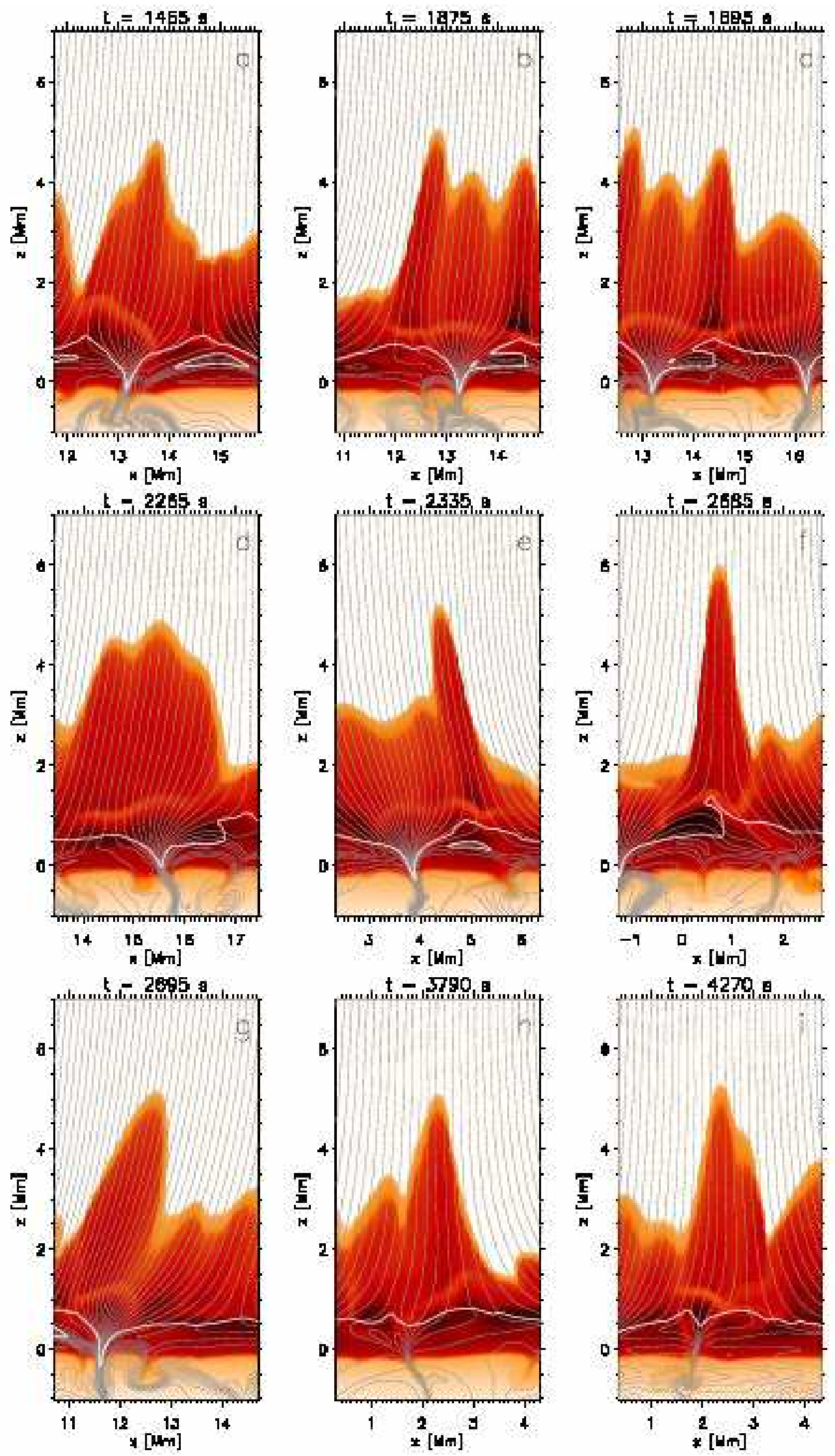

Figure 25. Temperature plot showing a selection of the tallest jets in case B. The gray lines are magnetic field lines, while the white line is at the height where $\beta=1$. 

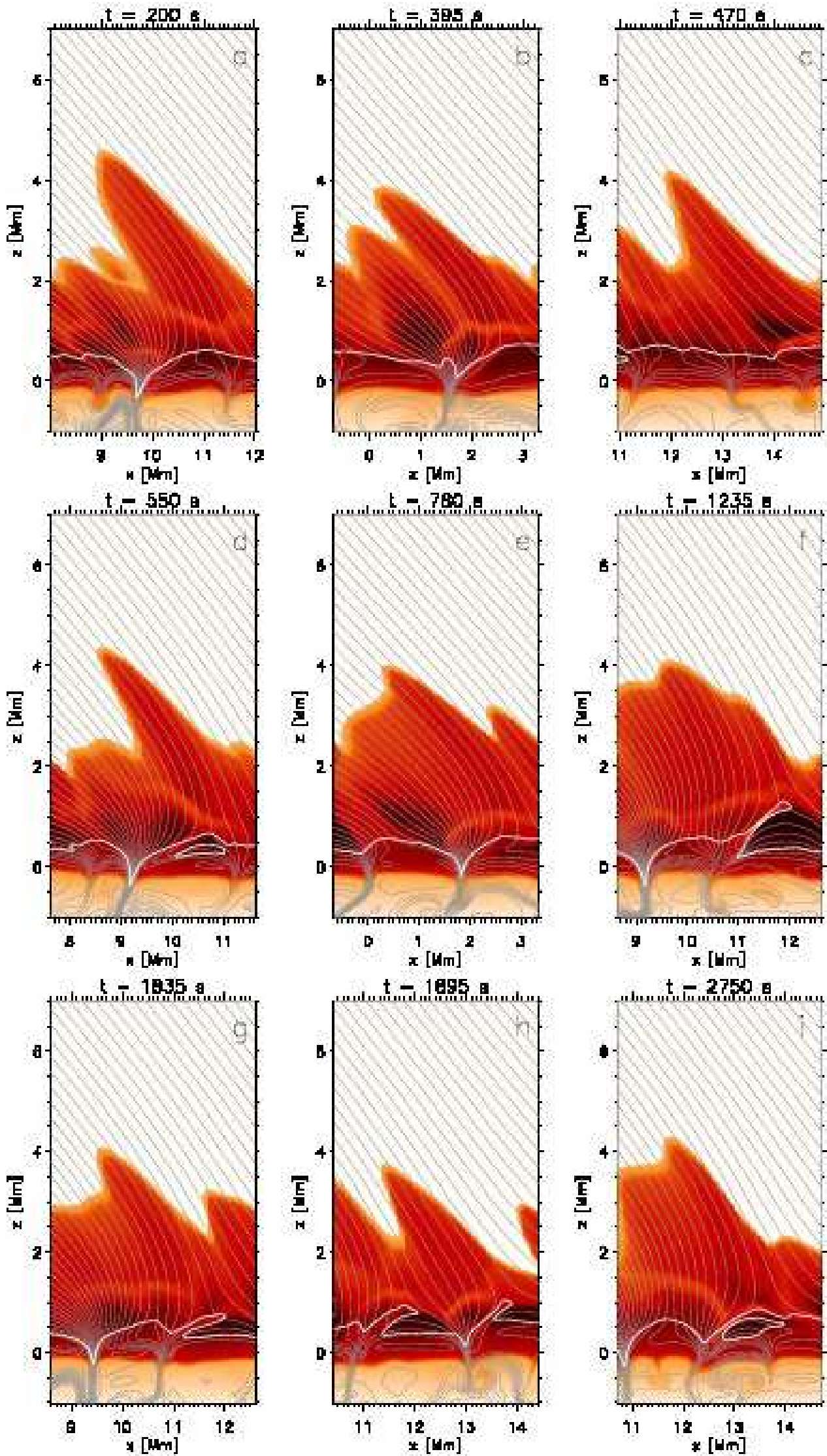

Figure 26. Temperature plot showing a selection of the tallest jets in case D. 

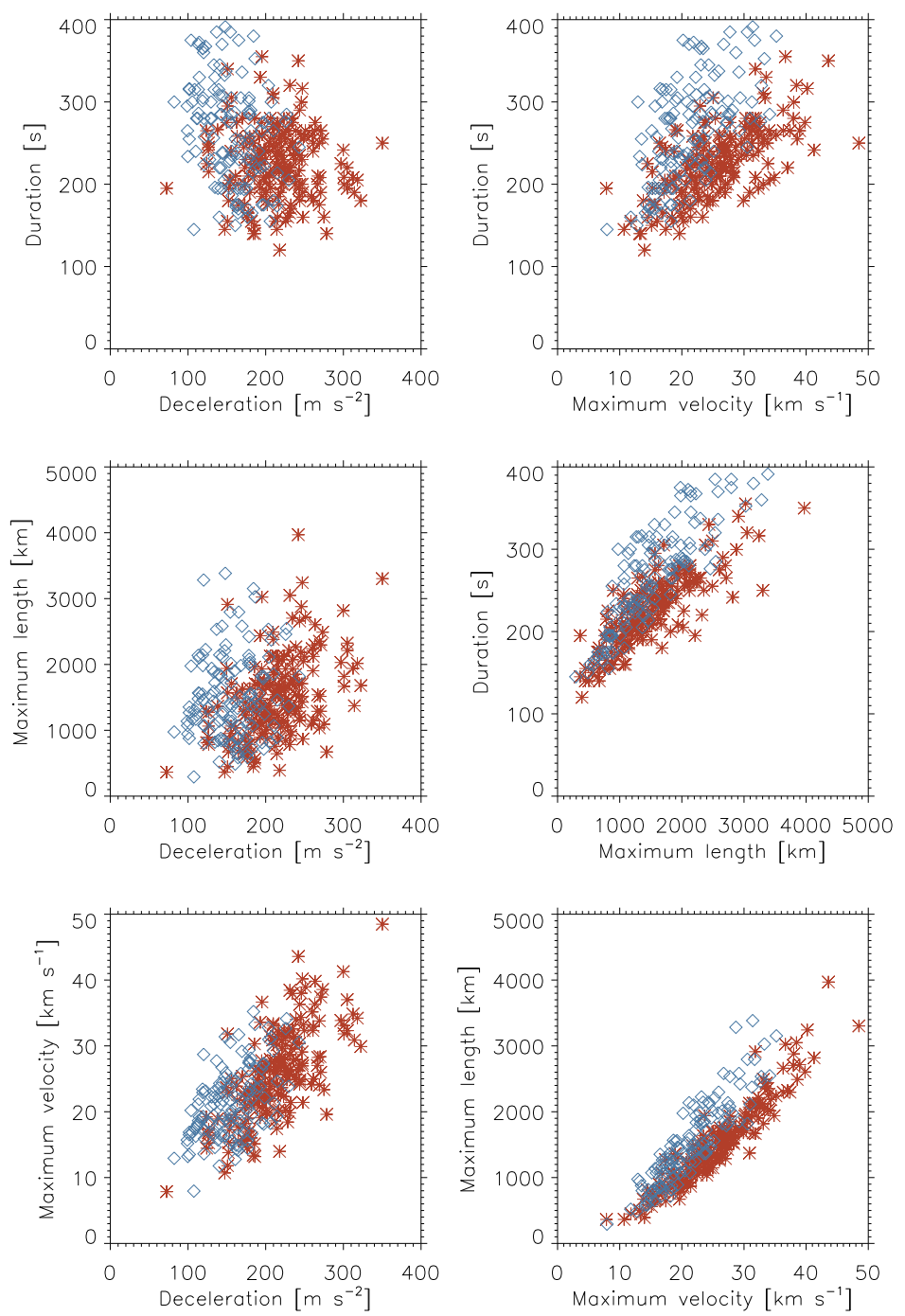

Figure 27. Scatter plots showing correlations between different jet properties in case B (vertical field, shown as red asterisks) and case D (inclined field, shown as blue diamonds). Most quantities exhibit linear correlations; a particularly nice one is the one between maximum velocity and maximum length (bottom right). There is however no strong correlation between the deceleration and either the duration (top left) or the maximum length (center left).

format as Figure 1 in Martínez-Sykora et al. (2009) for ease of comparison. They should also be compared with Figures 12 and 13 in De Pontieu et al. (2007).

Overall, the results of the different simulations and the observations are in good agreement. As we see, there are clear linear correlations between several of the jet properties, in particular between maximum velocity and maximum length (bottom right), deceleration and maximum velocity (bottom left), and maximum length and duration (center right). The same correlations are found in observations by De Pontieu et al. (2007), and in simulations by Heggland et al. (2007), who only plot deceleration vs. maximum velocity, and Martínez-Sykora et al. (2009), who find a weaker correlation between length and duration. In addition, we find a correlation between the maximum velocity and the duration (top right) in our simulations, whereas De Pontieu et al. and Martínez-Sykora et al. find little evidence of a correlation here. Conversely, they both find a weak anticorrela- tion between deceleration and duration (top left). In our simulations, there is a quite weak anticorrelation in the distribution for case D (blue diamonds), whereas case B (red asterisks) shows little evidence of a correlation. The two distributions do however cluster in different regions, with the longer-duration jets in case D having lower decelerations than the shorter-lived jets in case B. This regional difference between the distributions was also found in the observations. Neither we, Martínez-Svkora et al., nor De Pontieu et al. find any strong correlation between the deceleration and the maximum length of the jets (center left).

As noted above, the properties of the jets formed in case B and case D can be somewhat different - for example, at a given maximum velocity, the jets in case $\mathrm{D}$ (inclined field) tend to be longer (bottom right panel) and have a longer duration (top right panel). In order to further investigate these differences, we have made histograms showing the distributions of the jet properties 

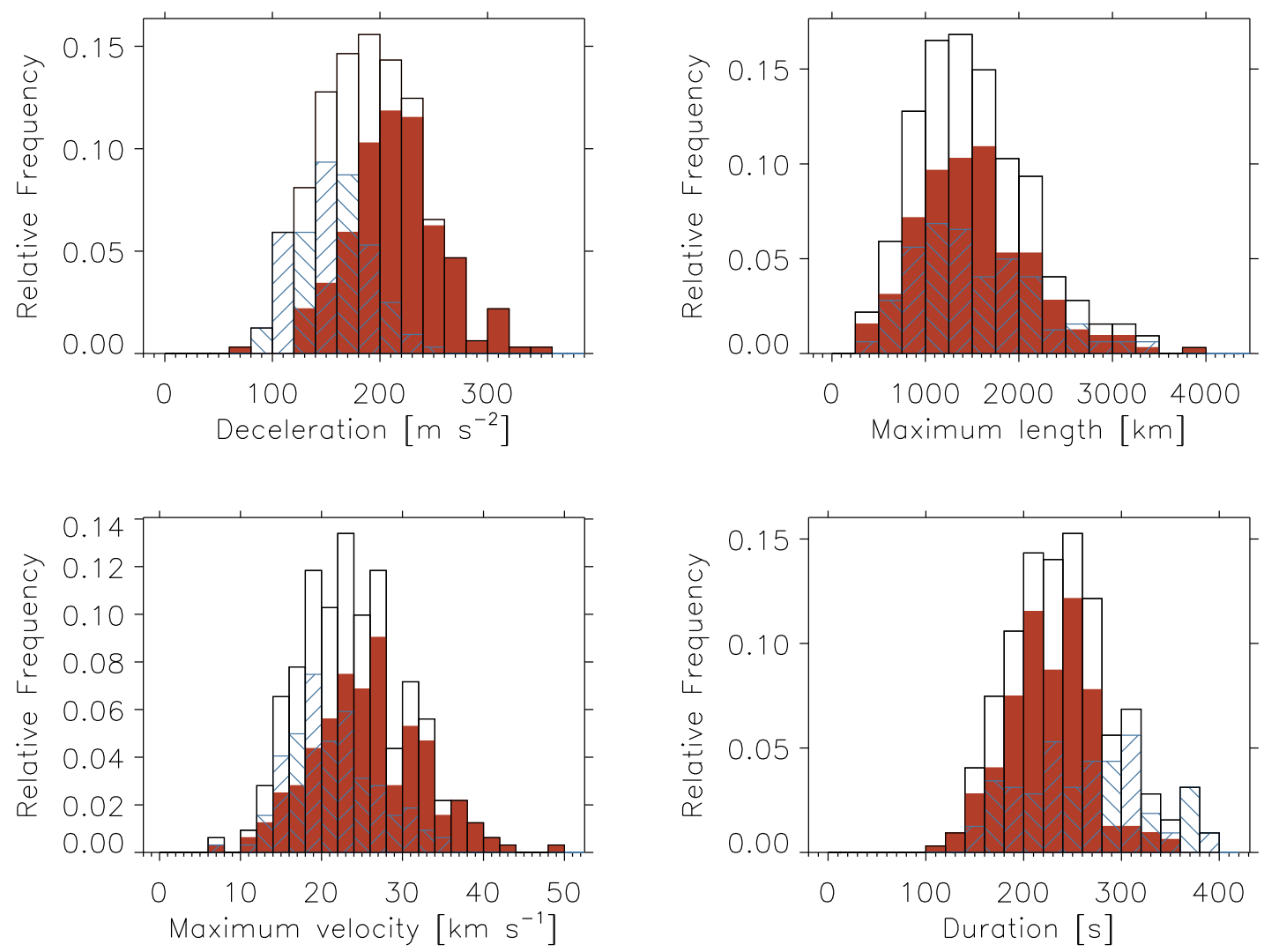

Figure 28. Histograms showing the distributions of deceleration (top left), maximum length (top right), maximum velocity (bottom left), and duration (bottom right). Case B (vertical field) is shown as red solid columns, case D (inclined field) is shown as blue hatched columns, and the sum is shown as black open columns.

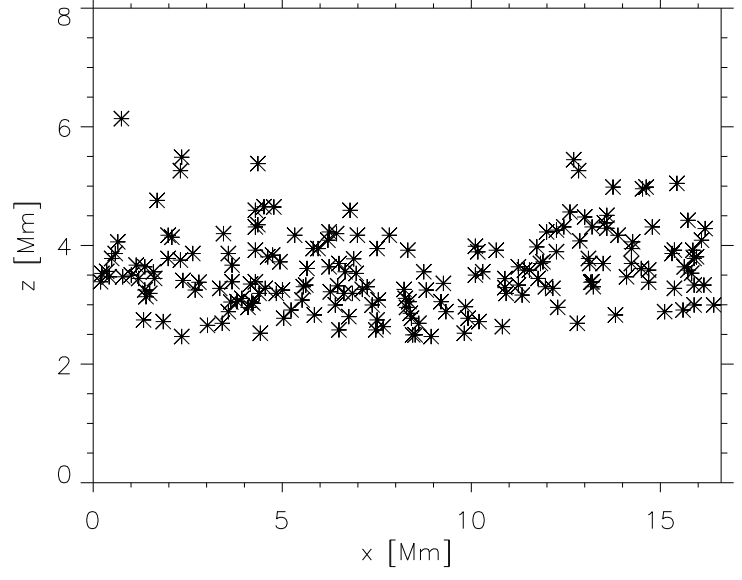

Figure 29. Scatter plot of jet heights versus horizontal position in case B.

in the two cases. The results are plotted in Figure 28, which shows the jets in case $\mathrm{B}$ as solid red columns, case $\mathrm{D}$ as hatched blue columns, and the sum as black outlines. The distributions are notably different with respect to deceleration (upper left panel) and duration (lower right), with the inclined jets lasting longer and having lower decelerations. The distributions of maximum lengths (upper right) are broadly similar, but the inclined jets have somewhat lower maximum velocities (lower left). De Pontieu et al. (2007) found similar regional differences in their observations, with jets in dense plage regions (referred to as region 2 in their paper) having higher decelerations and shorter lifetimes than jets in adjacent regions with weaker and more inclined field (referred to as region 1). They suggested that the differences were due to the field inclination, which is supported by our results. The results are also consistent with what we have found out about wave propagation in the different models: long-period waves are more prevalent in inclined field regions, and these waves then produce longer-lived jets with lower decelerations.

In addition to differences between the cases, there are also some regional differences within each case. We see in Figure 25 that a number of the tallest spicules in case $\mathrm{B}$ are located in regions where field lines from different flux concentrations meet (ref. Figure 9). This is particularly noticeable in the cases of jets $e$ and $f$, where the central axis is right along the interface region. Jets $b$, $c, h$, and $i$ are also located close to the interface, while jets $a$ and $g$ are above the center of their connected flux concentrations.

In Figure 29, we have plotted the maximum heights reached by the jets as a function of their horizontal location. Notably, the jets that form above the central, wide flux concentration $(x=8-11 \mathrm{Mm})$ reach lower heights than the ones forming in other locations, while the tallest 
jets form in interface regions between flux concentrations at $x=13-15 \mathrm{Mm}$ and $x=0-4 \mathrm{Mm}$. There are at least two possible explanations why this should be so. One is that you can get constructive interference between the waves coming from different flux concentrations, leading to stronger shocks and longer jets. For example, jet $e$ in Figure 25 can be traced to such an event happening at $x=5 \mathrm{Mm}, t=2150 \mathrm{~s}$ (cf. Figure 9, which shows the velocity field). Another possible explanation is that when several flux concentrations (of the same polarity) are located near each other, the field lines connected with each cannot spread out over such a large area, and the waves coming from below will be more concentrated and propagate more vertically. Conversely, above the large central flux concentration, the wave energy is spread over a much larger area as much of it follows the widening field. This then leads to wider but less powerful jets.

\section{SUMMARY}

We have presented several simulations investigating the periodicity of waves propagating through the chromosphere. We find that waves with periods of around 5 minutes can propagate in regions where the magnetic field is strong and inclined, including at the edges of flux tubes. In regions where the magnetic field is weak or vertical, we find primarily 3 -minute waves; this also applies above vertical flux tubes and in the center of strong expanding flux tubes. These results indicate that field inclination is critical to the propagation of long-period waves. Where the flux tubes undergo significant horizontal motion, both the 5 -minute and the 3 -minute power is spread out and the distinction is not as clearly visible. Since we have included an advanced treatment of radiative losses in our simulations and find 3-minute propagation above vertical field regions, we conclude that variation in the radiative relaxation time is not an effective mechanism for increasing the cutoff period. Our simulations are in agreement with the results of recent high-resolution Hinode observations.

We have also studied the jets produced by these waves once they reach the transition region. We find systematic differences between the jets produced in a model with mostly vertical field, and in a model with mostly inclined field. The results are in agreement with observations of dynamic fibrils.

It is also important to point out, for the purpose of future analyses of wave propagation and periodicity, whether from simulations or observations, that the Fourier analysis can be misleading and hide important information about the state of the medium. This is because the solar atmosphere is dynamic and changes on timescales much shorter than one hour, which is often the minimum timescale needed to achieve sufficient spectral resolution in a Fourier analysis. A wavelet analysis takes variations in time into account and can be an invaluable tool for figuring out what processes are important for the dynamics.
This work was supported by the Research Council of Norway through grant 159483/V30. Bart De Pontieu was supported by NASA grants NNX08AL22G and NNX08BA99G.

\section{REFERENCES}

Bel, N., \& Leroy, B. 1977, A\&A, 55, 239

Berger, T. E., \& Title, A. M. 1996, ApJ, 463, 365

Carlsson, M. 1986, Uppsala Astronomical Observatory Reports, 33

Carlsson, M., \& Stein, R. F. 1995, ApJ, 440, L29

Carlsson, M., \& Stein, R. F. 1997, ApJ, 481, 500

Centeno, R., Collados, M., \& Trujillo Bueno, J. 2006, ApJ, 640, 1153, arXiv:astro-ph/0512096

Centeno, K., Collados, $\bar{M}$., \& Trujillo Bueno, J. 2009, ApJ, 692, 1211, arXiv:astro-ph/0810.3613

De Pontieu, B., Erdélyi, R., \& De Moortel, I. 2005, ApJ, 624, L61

De Pontieu, B., Erdélyi, R., \& James, S. P. 2004, Nature, 430, 536

De Pontieu, B., Hansteen, V. H., Rouppe van der Voort, L., van Noort, M., \& Carlsson, M. 2007, ApJ, 655, 624

de Wijn, A. G., McIntosh, S. W., \& De Pontieu, B. 2009, ApJ, 702, L168, arXiv:0908.1383

Erdélyi, R., Malins, C., Tóth, G., \& De Pontieu, B. 2007, A\&A, 467, 1299

Giovanelli, R. G., Livingston, W. C., \& Harvey, J. W. 1978, Sol. Phys., 59, 49

Gudiksen, B. V., Carlsson, M., Hansteen, V. H., Hayek, W., Leenaarts, J., \& Martínez-Sykora, J. 2011, A\&A, 531, A154

Hansteen, V. H., De Pontieu, B., Rouppe van der Voort, L., van Noort. M.. \& Carlsson. M. 2006, ApJ, 647, L73, arXiv:astro-ph/0607332

Hayek, W. ., Aspiund, IVi., Carlsson, M., Trampedach, R., Collet, R., Gudiksen, B. V., Hansteen, V. H., \& Leenaarts, J. 2010, A\&A, 517, A49

Heggland. L.. De Pontieu. B.. \& Hansteen, V. H. 2007, ApJ, 666, 1277, arXiv:astro-ph/0703498

Jefferies, S. ivi., Ivicintosh, S. W. Armstrong, J. D., Bogdan, T. J., Cacciani, A., \& Fleck, B. 2006, ApJ, 648, L151

Judge, P. G., \& Meisner, R. 1994, in The Third Soho Workshop, Solar Dynamic Phenomena and Solar Wind Consequences, ed. J. J. Hunt (Noordwijk: ESA SP-373), 67

Kato, Y., Steiner, O., Steffen, M., \& Suematsu, Y. 2011, ApJ, 730, L24, arXiv:1102.5164

Khomenko, E., Centeno, R., Collados, M., \& Trujillo Bueno, J. 2008, ApJ, 676, L85, arXiv:astro-ph/0802.0938

Leenaarts, J., Carlsson, M., Hansteen, V., \& Gudiksen, B. V. 2011, A\&A, 530, A124, arXiv:1104.5081

Leenaarts, J., Carlsson, M., Hansteen, V., \& Rutten, R. J. 2007 A\&A, 473, 625, arXiv:astro-ph/0709.3751

Lites, B. W., Rutten, R. J., \& Kalkofen, W. 1993, ApJ, 414, 345

Martínez-Sykora, J., Hansteen, V., De Pontieu, B., \& Carlsson,

M. 2009, ApJ, 701, 1569, arXiv:0906.4446

Michalitsanos, A. G. 1973, Sol. Phys., 30, 47

Nordlund, A. 1982, A\&A, 107, 1

Orrall, F. Q. 1966, ApJ, 143, 917

Roberts, B. 1983, Sol. Phys., 87, 77

Rouppe van der Voort, L. H. M., De Pontieu, B., Hansteen, V. H., Carlsson, M.. \& van Noort, M. 2007, ApJ, 660, L169, arXiv:astro-ph/0703535

Schmitz, F., \& Fleck, B. 1998, A\&A, 337, 487

Skartlien, R. 2000, ApJ, 536, 465

Stangalini, M., Del Moro, D., Berrilli, F., \& Jefferies, S. M. 2011, A\&A, 534, A65, arXiv:1108.4576

Sterling, A. C. 2000, Sol. Phys., 196, 79

Suematsu, Y. 1990, in Proc. Oji International Seminar, Progress of Seismology of the Sun and Stars, ed. Y. Osaki \&

H. Shibahashi, (LNP, Vol. 367; Berlin: Springer), 211

Torrence, C., \& Compo, G. P. 1998, Bull. Am. Meteorol. Soc., 79, 61

Vernazza, J. E., Avrett, E. H., \& Loeser, R. 1981, ApJS, 45, 635 